Bundesgesundheitsbl 2017 · 60:1356-1371 https://doi.org/10.1007/s00103-017-2641-7 Online publiziert: 24. Oktober 2017 (c) Der/die Autor(en) 2017. Dieser Artikel ist eine Open-Access-Publikation.

CrossMark

Dominik von Stillfried - Tatiana Ermakova - Frank Ng - Thomas Czihal

Zentralinstitut für die kassenärztliche Versorgung, Berlin, Deutschland

\title{
Virtuelle Behandlernetzwerke
}

\section{Neue Ansätze zur Analyse und Veränderung räumlicher Versorgungsunterschiede}

\section{Hintergrund}

Ausgehend von Wennberg und Gittelsohn (1973) hat sich die Analyse räumlicher Unterschiede in der medizinischen Versorgung weltweit als Forschungsrichtung etabliert [1]. Sie fokussiert auf die Frage, was die medizinische Versorgung für die Menschen unter Alltagsbedingungen leistet. Die geografische Betrachtung vereinfacht es, über den Wohnort der Patienten einen Populationsbezug herzustellen, um etwa standardisierte Eingriffshäufigkeiten oder Behandlungserfolge vergleichen zu können.

Die Fülle verfügbarer Publikationen zeigt, dass Ausmaß und Persistenz der dokumentierten räumlichen Versorgungsunterschiede weitgehend unabhängig von organisatorischen oder regulativen Rahmenbedingungen der einzelnen Gesundheitssysteme bestehen. Sie stellen nach Einschätzung der Organisation für wirtschaftliche Zusammenarbeit und Entwicklung (OECD) eine enorme gesundheitspolitische Herausforderung dar [2]. Unbegründete Versorgungsunterschiede widersprechen aber dem Anspruch der Medizin nach größtmöglicher Effektivität. Der Zugang zu medizinischer Versorgung gilt in den Industrieländern als wesentlicher Teil staatlich verantworteter Daseinsvorsorge. Die damit verbundenen Gerechtigkeitsziele werden durch ausgeprägte regionale Versorgungsunterschiede potenziell infrage gestellt. Zudem werfen sie die Frage auf, welche Versorgungsintensität zur Wahrung der Effizienzziele der sozialen Sicherungssysteme ausreichend bzw. als Überversorgung einzustufen ist.
Räumliche Versorgungsanalysen zielen daher meist darauf ab, Bewusstsein für notwendige Veränderungen im Versorgungsalltag zu schaffen und Anhaltspunkte zum Abbau unerklärter bzw. unerwünschter Versorgungsunterschiede zu finden. Nach dem Vorbild des Dartmouth Atlas of Healthcare sind daher in vielen Ländern interaktive Atlanten der medizinischen Versorgung entstanden, die das Handeln der Entscheidungsträger verändern oder beeinflussen sollen. ${ }^{1}$

$\mathrm{Da}$ in den meisten steuerfinanzierten Gesundheitssystemen administrative Einheiten mit geografisch definierten Zuständigkeiten für Gestaltung und Qualität der Versorgung existieren, kann die räumliche Analyse hier Unterstützung bieten. In Versicherungssystemen mit freier Arztwahl können geografische Unterschiede in der Versorgung nicht ohne weiteres mit dem Verhalten einzelner Praxen oder Krankenhäuser in eine direkte Verbindung gebracht werden. Im deutschen Gesundheitssystem wird dies z. B. durch die unterschiedlich stark ausgeprägten überregionalen Mitversorgungsfunktionen verdeutlicht [3]. Um die Bedeutung unterschiedlicher Behandlungsmuster für die jeweils versorgte Bevölkerung kartografisch darstellen zu können, konstruiert der DartmouthAtlas daher Wirkungsbereiche für Krankenhäuser und Arztpraxen (z. B. „hos-

\footnotetext{
1 Vgl. z. B. https://www.england.nhs.uk/ rightcare/; https://www.hqsc.govt.nz/ourprogrammes/health-quality-evaluation/ projects/atlas-of-healthcare-variation/; www. versorgungsatlas.de; https://faktencheckgesundheit.de; sowie eine internationale Übersicht der Forschungsaktivitäten hierzu: http:// wennbergcollaborative.org/publications. Zugegriffen:31.Juli 2017.
}

pital referral regions“, „hospital service areas", „primary care service areas" [4]).

Die Analyseergebnisse werden insoweit als regionale Mittelwerte von Versorgungsindikatoren (z. B. Impfquoten) präsentiert. Unklar bleibt, wie aussagefähig solche Mittelwerte für Patienten und Versorger sein können. Um die eigene Praxis mit dem regionalen Mittelwert vergleichen zu können, bietet der neuseeländische Versorgungsatlas Hausarztpraxen daher eine Zusatzfunktion (,find my patients"), mit der diese analoge Auswertungen in der eigenen Praxis durchführen können (vgl. z. B. die Darstellung der Versorgung von Patienten mit Gicht im neuseeländischen Gesundheitsatlas [5]). Die Aussagefähigkeit der praxisinternen Analysen beruht jedoch darauf, dass Patienten in Neuseeland ohne Überweisung nicht zugleich mehrere Ärzte aufsuchen können.

Angesichts der Bedeutungszunahme chronisch behandlungsbedürftiger Krankheiten und fortschreitender Spezialisierung in der Medizin wird in den meisten Gesundheitssystemen nach Wegen zur Verbesserung der Kooperation und Koordination der Behandlungsprozesse gesucht [6]. Diese Zielsetzung gestaltet sich besonders komplex in $\mathrm{Ge}$ sundheitssystemen, in denen Patienten im Rahmen freier Arztwahl durch ihre Inanspruchnahme entscheiden, welche Ärzte, Praxen oder Krankenhäuser zur erfolgreichen Behandlung eines Patienten zusammenarbeiten sollten. In diesem Kontext ist davon auszugehen, dass die Verantwortung für die Behandlung vieler Patienten zwischen den an der Versorgung beteiligten Institutionen in unterschiedlichem Maße geteilt wird. 
Diese geteilte Verantwortung kann in virtuellen Behandlernetzwerken, sog. "patient-sharing networks" (PSN), abgebildet werden. Die faktische Verbundenheit der Behandler über ihre Patienten kann insbesondere auf der Grundlage von Abrechnungsdaten sichtbar gemacht werden. In den vergangenen Jahren ist hierzu ein wachsender Forschungszweig entstanden. Entsprechende Analysen sollen in den USA und Kanada auch dazu dienen, die Bildung von Accountable-Care-Organisationen (ACO) zu unterstützen, in denen die Koordination von Behandlungsprozessen gezielt weiterentwickelt werden soll.

Der Gedanke lässt sich auch auf das deutsche Gesundheitssystem übertragen, in dem Patienten größte Wahlfreiheiten genießen. Nicht zuletzt aufgrund der hohen Zahl von Arztkontakten [7, 8] sowie der zunehmenden Krankenhausaufnahmen ohne ärztliche Einweisung (vgl. z. B. $[9,10])$ steht in der Gesundheitspolitik und in der Versorgungsforschung die Frage im Vordergrund, wie die Koordination der Versorgung verbessert werden kann (vgl. z. B. [11]). Nachfolgend werden daher Methoden zur Abbildung von PSN im Hinblick darauf untersucht, welche Beiträge zur Erklärung räumlicher Behandlungsmuster diese leisten können bzw. inwieweit diese Interventionen zur Verringerung unerwünschter Unterschiede in der Versorgung unterstützen können.

\section{Suche nach Methoden zur Abbildung virtueller Netzwerke}

Ausgehend von der Annahme, dass PSN sich in dezentralen Selbstorganisationsprozessen bilden, werden Methoden gesucht, die eine sinnvolle Abgrenzung bzw. Abbildung der virtuellen Netzwerke unter folgenden Gesichtspunkten ermöglichen:

- Information der in der Versorgung tätigen Einrichtungen über Art und Ausmaß ihrer Verbundenheit mit anderen Einrichtungen,

- Identifikation der gemeinsam versorgten Patientenpopulation und der Versorgungsergebnisse für die Population,
- Unterscheidung zwischen einer vom Versorger ungesteuerten Verbundenheit infolge patientenseitiger Inanspruchnahmeprozesse und einer vom Versorger beabsichtigten Kooperation,

- Weiterentwicklung virtueller Netzstrukturen zu Kooperationsstrukturen in der medizinischen Versorgung.

Nachfolgend werden Zwischenergebnisse einer Suche nach einem neuen Instrumentarium dargestellt, auf dessen Grundlage Interventionen zur Veränderung komplexer Versorgungsabläufe unter Beteiligung mehrerer Praxen und/ oder Krankenhäuser unter Alltagsbedingungen ermöglicht werden sollen. Im Rahmen dieser Suche sind sowohl die zu beantwortenden Fragestellungen als auch die eingesetzten Methoden in Bewegung. Um dieses sich entwickelnde Feld zu beschreiben, umfasst dieser Beitrag sowohl eine Literaturübersicht als auch einen knappen Werkstattbericht $\mathrm{zu}$ eigenen bisher unveröffentlichten Arbeiten. Die Autoren hoffen, dass die hierdurch gegebenen Anregungen zu weiteren Forschungsarbeiten auf diesem Gebiet die Unschärfen kompensieren, die durch die Kombination beider Formate unvermeidlich sind.

\section{Stand der internationalen Forschung}

Es wurde bisher keine systematische Literaturrecherche durchgeführt. Sucht man über das Kriterium „patient-sharing network" in den Datenbanken des National Center for Biotechnology Information (NCBI) [12] und von ResearchGate [13], stößt man auf 5 bzw. weitere 10 relevante Veröffentlichungen. Über das Kriterium "patient-sharing" ergeben sich bei NCBI 18 relevante Literaturstellen. Insgesamt ergeben sich über beide Quellen $19 \mathrm{Re}$ ferenzen. 15 weitere Quellen waren den Autoren aus persönlicher Kommunikation oder als zitierte Literatur bekannt. Die nachfolgende Übersicht versucht, dieses noch junge, heterogene Forschungsfeld anhand ausgewählter Referenzen zu beschreiben.

Publizierte Forschungsarbeiten zur Thematik finden sich fast ausschließlich in Nordamerika. Diese gliedern sich in instrumentelle und analytische Ansätze. Während instrumentelle Ansätze auf eine Intervention zielen und hierfür normative Festlegungen treffen, welche Akteure im Zentrum der zu betrachtenden PSN stehen, zielen analytische Ansätze primär auf Beschreibung und Verständnis empirischer Vernetzungsprozesse $a b$, ohne Rücksicht darauf, ob sich die daraus ergebenden Netzstrukturen für spätere Interventionen eignen.

Instrumentelle Ansätze/normative Festlegung eines Netzmittelpunkts

Um die Bildung künftiger ACOs zu fördern, haben Bynum et al. PSN beschrieben, die distinkte Patientenpopulationen versorgen und in deren Mittelpunkt jeweils ein Krankenhaus steht [14]. Patienten werden jeweils dem Arzt zugeordnet, der von ihnen am stärksten in Anspruch genommen wurde („usual provider“). Die Ärzte und deren Patienten wiederum werden dem Krankenhaus zugeordnet, mit dem die Ärzte jeweils direkt („hospital privileges“) oder indirekt (,referral“) am stärksten verbunden sind. Wie Landon et al. hervorheben, sollen bereits existierende Kooperationsbeziehungen identifiziert werden, die sich als Grundlage für die Bildung von ACOs eignen könnten [15]. Stukel et al. berichten über ein vergleichbares Vorgehen, um sog. "multispecialty physician networks“ in der kanadischen Provinz Ontario abzubilden [16]. Bei der Zuordnung des Patienten zum „usual provider“ steht das Inanspruchnahmeverhalten der Patienten im Vordergrund, bei der Zuordnung des Arztes zu einem Krankenhaus die Kooperationsbeziehung der jeweiligen Ärzte mit bestimmten Krankenhäusern. Zielsetzung hierbei ist, die Behandlungskette in der Versorgung chronisch Kranker und die faktischen Funktionsbeziehungen der Krankenhäuser mit den niedergelassenen Ärzten im Raum möglichst vollständig abzubilden. Gesundheitspolitisch zielt das Vorgehen auf eine Verringerung von Wiederaufnahmen nach stationärer Behandlung für bestimmte chronische Krankheiten ab. Deshalb wurde auch die Stabilität der Kooperationsbeziehungen im Zeit- 
Bundesgesundheitsbl 2017·60:1356-1371 https://doi.org/10.1007/s00103-017-2641-7

(c) Der/die Autor(en) 2017. Dieser Artikel ist eine Open-Access-Publikation.

\author{
D. von Stillfried · T. Ermakova · F. Ng · T. Czihal
}

\title{
Virtuelle Behandlernetzwerke. Neue Ansätze zur Analyse und Veränderung räumlicher Versorgungsunterschiede
}

\section{Zusammenfassung}

Damit auf unerklärte und unerwünschte regionale Unterschiede der Gesundheitsversorgung gezielt reagiert werden kann, ist es notwendig, die beobachteten Befunde dem Handeln der einzelnen Akteure (Patienten, Ärzte, Krankenhäuser) zuzuordnen. Die verschiedenen Akteure teilen sich in der Regel die Verantwortung für die Versorgungsprozesse und Behandlungserfolge einer Patientenpopulation in virtuellen Behandlernetzwerken („,patient-sharing network", PSN). Art und Umfang der gemeinsamen Behandlung lassen sich anhand von Abrechnungsdaten nachvollziehen. Ein neues Forschungsfeld befasst sich mit der
Darstellung der Behandlernetzwerke und deren Bedeutung für die Versorgung. Der Beitrag gibt einen Überblick, mit welchen Methoden die Netzwerke bisher dargestellt und analysiert wurden und inwieweit ihre Erforschung eine Grundlage für konkrete Maßnahmen zur Verbesserung der Gesundheitsversorgung liefern kann. Bisherige Ergebnisse legen nahe, dass nicht primär der Wohnort, sondern Art und Umfang der Kooperation in der Versorgung über den Behandlungserfolg entscheiden. Netzwerktheoretische Methoden (,social network analysis") können die epidemiologisch geprägten Verfahren der räumlichen Versorgung sinnvoll ergänzen. Methodische Fragen zur Abgrenzung relevanter Vernetzungspartner und deren Patientenpopulation sind noch zu klären. Erste Projekte nutzen PSN auch als Interventionsebene, um gezielte Kooperationen zur Verringerung unerwünschter regionaler Versorgungsprozesse herbeizuführen.

Schlüsselwörter

Kleinräumige Versorgungsunterschiede . Funktionale Populationen - Behandlernetzwerke - Analyse sozialer Netzwerke Graphentheorie · Routinedatenanalyse

\section{Patient-sharing networks. New approaches in the analysis and transformation of geographic variation in healthcare}

\section{Abstract}

The analysis of geographic variations has spurred arguments that area of residence determines access to and quality of healthcare. In this paper we argue that unwarranted geographic variations can be traced back to actions of individual patients and their healthcare providers (doctors, hospitals). These actors interact in a complicated web of shared responsibilities. Designing effective interventions to reduce unwarranted geographic variations may therefore depend on methods to identify these interactions and communities of providers with a shared accountability. In the US, Canada, and Germany, routine data have been used to identify self-organized informal or virtual networks of physicians and hospitals, socalled patient-sharing networks (PSNs). This is an emerging field of analysis. We attempt to provide a brief report on the state of work in progress. It can be shown that variation between PSNs in a given area is effectively greater than variation between regions. While this suggests that reducing unwarranted variation needs to start at the level of PSN, methods to identify PSNs still vary widely. We compare epidemiological approaches and approaches based on graph theory and social network analysis. We also present some preliminary findings of exploratory analyses based on comprehensive claims data of physician practices in Germany. Defining PSNs based on usual provider relationships helps to create distinctive patient populations while PSNs may not be mutually exclusive. Social network analysis, on the other hand, appears better equipped to differentiate between provider communities with stronger and weaker ties; it does not yield distinctive patient populations. To achieve accountability and to support change management, analytic methods to describe PSNs still need refinement. There are first projects in Germany which use PSNs as an intervention platform in order to achieve improved cooperation and reduce unwarranted variation in their care processes.

\section{Keywords}

Geographic variation in healthcare · Functional populations - Patient-sharing networks - Social network analysis - Graph theory · Analysis of claims data verlauf beachtet. Um stabile Netzwerke $\mathrm{zu}$ erhalten, wurden teilweise mehrere Krankenhäuser und deren ambulante Kooperationspartner zusammengefasst. Hierdurch entstehen relativ große (5bis 6-stellige) Patientenpopulationen. Vergleiche über indikationsbezogene Behandlungsmuster werden so erleichtert. Da die gebildeten Netzwerke sehr unterschiedliche und teils überlappende räumliche Ausdehnungen aufweisen, ersetzt diese Herangehensweise den bis- herigen geografischen Vergleich nach Wohnregionen. Allerdings fallen nicht alle beobachteten stationären Behandlungen in die für das Netzwerk prägende Kooperationsbeziehung; die „Netzwerk-

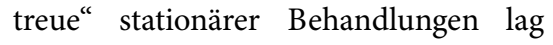
zwischen 30 und $70 \%$ je PSN.

Wer hingegen die primärärztliche Koordinationsfunktion stärken will, stellt nicht das Krankenhaus, sondern die Hausarztpraxis in den Mittelpunkt der Betrachtung. Pham et al. fokussieren auf die Frage, mit wie vielen anderen Ärzten bzw. Einrichtungen der Hausarzt seine Patienten teilt, um den Koordinationsaufwand in der Behandlung von staatlich krankenversicherten Patienten (Medicare-Patienten) zu verdeutlichen [17]. Anzahl und Struktur der mitbehandelnden Ärzte, speziell der Fachärzte („specialists“), weisen grundsätzliche Unterschiede auf, die teils auf den Standort, teils auf die Ausrichtung der Hausarztpraxen zurückgeführt werden kann. 
So ist die Zahl mitbehandelnder Ärzte in städtischen Regionen systematisch höher als in ländlichen und unterscheidet sich nach Bundesstaaten. Einzelpraxen, kleine Gemeinschaftspraxen und Praxen mit einem höheren Anteil chronisch Kranker sind in größere Netzstrukturen eingebunden als große Gruppenpraxen und Praxen mit wenig multimorbiden Patienten. Die Autoren sehen ihre Arbeit als mögliche Grundlage für eine Förderung gezielter Vernetzungsprozesse zur Stärkung des Prinzips von „medical homes“ („medizinischen Versorgungsteams"). Dieses sieht eine enge, technisch unterstützte und auf Langzeitbegleitung ausgerichtete Zusammenarbeit der Akteure vor, die vom Hausarzt koordiniert wird.

Pollack et al. verfolgen diesen Ansatz weiter und untersuchen den Effekt fachübergreifender Zusammenarbeit auf die Hospitalisierungsrate und den $\mathrm{Be}$ handlungsaufwand insgesamt [18]. Bereits in der Fragestellung wird deutlich, dass in einem solchen Ansatz möglichst alle stationären Aufenthalte - unabhängig von der Intensität der Kooperationsbeziehung einzelner Krankenhäuser mit niedergelassenen Ärzten - als Ergebnisvariable einzubeziehen sind. Folglich fokussiert die Analyse auf die faktische Vernetzung zwischen Primär- und ausgewählten Fachärzten, die für je eine Patientenkohorte (Herzinsuffizienz, Diabetes) als relevant definiert wurden. Die Autoren ermitteln anhand von Abrechnungsdaten mehrerer privater Versicherungsträger in den USA Indexwerte für die Zahl der ambulant tätigen Mitbehandler je Patient („care density“). Hohe Werte werden nach Darstellung der Autoren für Patienten erreicht, die kooperationsbereitere Ärzte aufgesucht haben. Für diese Patienten werden günstigere Outcomes (niedrigere Hospitalisierungsraten, geringere Ausgaben) berichtet [18]. Die Autoren sehen dies als Hinweis auf kooperativeres Verhalten der beteiligten Ärzte, allerdings wurden keine spezifischen Kooperationsmerkmale (etwa Art und Umfang der Kommunikation zwischen den beteiligten Ärzten) erhoben. Auch lässt die Datengrundlage keine patientenvollständige Analyse der jeweiligen Praxen zu, sodass bei die- ser Herangehensweise Schlussfolgerungen auf das Arztverhalten nur mit Einschränkung möglich sind. Unklar bleibt auch, welcher Einfluss auf die untersuchten Outcomes auf diejenigen Ärzte zurückzuführen ist, die von den betrachteten Patienten auch noch aufgesucht, aber in der Studie nicht berücksichtigt wurden, da per definitionem nur Ärzte bestimmter Fachgruppen berücksichtigt wurden.

Ein weiteres Beispiel für die indikationsspezifische Betrachtung ärztlicher Kooperation als Funktion von „patientsharing" bieten Hussain et al. [19]. Sie sehen in der Abrechnung von Leistungen für denselben Patienten Hinweise auf Kooperationsbeziehungen („advice seeking and referral relationships") zwischen Fachärzten und untersuchen den Effekt von „patient-sharing“ zwischen Chirurgen und Onkologen auf die Überlebensrate einer Kohorte von Kolonkarzinompatienten. Eine höhere Rate von "patient-sharing“ ist mit höheren 10Jahres-Überlebensraten der Patienten assoziiert. Auch hier bleibt aufgrund des gewählten Datenausschnitts und der normativen Eingrenzung der zu untersuchenden Kooperationsbeziehung unklar, ob ggf. weitere Einflüsse gemeinsam auf den Vernetzungsgrad und die Überlebensrate wirken.

\section{Analytische Ansätze zur Identifikation von Gemeinschaften in Netzwerken}

Mit Hilfe von graphentheoretischen Analyseansätzen können soziale Netzwerke untersucht, Gemeinschaften von Akteuren (Communities) erkannt und voneinander abgegrenzt werden (vgl. z. B. [20, 21]). Soziale Netzwerke werden - ähnlich wie bspw. technologische oder biologische Netzwerke - abstrakt als eine Menge von Akteuren (auch: Knoten, „nodes") und eine Menge von Verbindungen (auch: Kanten, „edges“) zwischen den Akteuren beschrieben. Anhand der Verbindungen zwischen Akteuren können Netzwerkstrukturen sowie die Bedeutung einzelner Akteure (Zentralität, „centrality“) für eine oder mehrere Gemeinschaften (Communities) beschrieben werden. Die Graphentheorie bietet zahlreiche Kennzahlen bzw. Metriken zur Beschreibung von sozialen Netzwerken, wovon hier 4 Metriken kurz erläutert werden sollen: So beschreibt etwa die Gradzentralität („degree centrality“) die Anzahl der Verbindungen eines Akteurs zu anderen Akteuren. Die Nähezentralität („closeness centrality“) misst den kürzesten Weg (Pfaddistanz) eines Akteurs zu allen anderen Akteuren in einem Netzwerk und bestimmt so die Zentralität für den Akteur. Die Zwischenzentralität („betweenness centrality“) hingegen misst die Anzahl, wie oft ein Akteur auf den kürzesten Wegen zwischen weiteren Akteuren liegt. Schließlich kann die Eigenvektorzentralität („eigenvector centrality") als Erweiterung der Gradzentralität verstanden werden, die die Zentralität von Nachbarakteuren mit einbezieht. Die Eigenvektorzentralität folgt damit dem Gedanken, dass die Zentralität eines Akteurs auch von der Zentralität seiner Nachbarn abhängt [22].

Anhand der Zentralitätsmetriken können Eigenschaften der Netzwerkstrukturen beschrieben werden. Dies ermöglicht zudem, dass die Akteure eines Netzwerkes miteinander verglichen werden können, sodass sie bzgl. ihrer Ähnlichkeit im Hinblick auf die untersuchten Merkmale (Homophilie) dargestellt werden können. Schließlich bietet die Graphentheorie zahlreiche Instrumente, um Gemeinschaften von Akteuren („communities“) innerhalb großer Netzwerke voneinander abzugrenzen. So wird bspw. der Modularitätswert („modularity“) herangezogen, um Teilnetzwerke hinsichtlich ihrer inneren Kohärenz und gleichzeitig der Trennschärfe zu anderen Teilnetzwerken zu bewerten. Der Modularitätswert gibt die Anzahl der Verbindungen innerhalb der Teilnetzwerke abzüglich einer erwarteten Anzahl an Verbindungen in einem äquivalenten Netzwerk mit einer durchschnittlichen Verteilung der Verbindungen wieder. Somit ist der Modularitätswert umso höher, je höher die tatsächliche Anzahl der Verbindungen im Vergleich $\mathrm{zu}$ den $\mathrm{zu}$ erwartenden Verbindungen ausfällt. Die präzise mathematische Berechnungsformel findet sich zum Beispiel bei Newman [23, S. 8578]. 
Moen et al. (2016) [24] wenden in einer Fallstudie das netzwerktheoretische Analyseinstrumentarium auf die von Bynum et al. [14] gebildeten krankenhauszentrierten Netzwerke an, um Eigenschaften zu bestimmen, die eine Leitlinienadhärenz bei der Implantierung von Defibrillatoren unterstützen. Sie suchen nach Faktoren zur Gewichtung der Verbindungen zwischen Ärzten und Krankenhäusern und zwischen den Ärzten innerhalb eines krankenhauszentrierten Netzwerks. Untersucht wird etwa, inwieweit die Zentralität einzelner Krankenhäuser innerhalb des Netzwerks („,betweenness centrality") oder die Vernetzung eines netzwerkzentralen Krankenhauses mit den Krankenhäusern anderer Netzwerke („closeness centrality“) Einfluss auf die Leitlinienadhärenz haben. Während für Zentralitätsmerkmale der Krankenhäuser und für die Fachgruppenzusammensetzung (Homophilie) der PSN kein Einfluss nachgewiesen werden kann, wird erkennbar, dass der Intensitätsgrad der Vernetzung zwischen Ärzten eines PSN („degrees“) mit der Größe des Netzwerks abnimmt. Dabei erwies sich die Zentralität der für die Durchführung der Implantation maßgeblichen Fachärzte innerhalb der krankenhauszentrierten PSN als signifikanter Einflussfaktor, um die zwischen zwei benachbarten Krankenhausmärkten („hospital referral regions") beobachteten Behandlungsunterschiede (Leitlinienadhärenz) zu erklären. Die Autoren schließen daraus, dass die Methoden der sozialen Netzwerkanalyse auch zum besseren Verständnis räumlicher Versorgungsunterschiede beitragen können.

Soziale Netzwerke haben in der Regel kaum räumliche oder andere natürliche Grenzen. Einerseits weisen Beziehungen zwischen Akteuren an bestimmten Stellen Verdichtungen (Cluster) auf, die als Teilnetzwerke bezeichnet werden können, andererseits bestehen in der Regel Verbindungen einzelner Akteure zu Akteuren anderer Cluster bzw. Teilnetzwerke. Um in dieser Komplexität verbundener Akteure einzelne Gemeinschaften („communities detection“) abgrenzen zu können, die in der Regel mehrere Akteure umfassen, werden auch besondere graphentheoretische Analyseansätze an- gewendet. Diese suchen - auch mithilfe der Wahrscheinlichkeitstheorie - unter der Vielzahl möglicher Konstellationen eine „beste“ Einteilung der verfügbaren Akteure in sinnvolle Gemeinschaften bzw. Teilnetzwerke [25, 26]. Hierfür existieren unterschiedliche Algorithmen, die in unterschiedlicher Weise auf die oben beschriebenen Zentralitätsmaße aufsetzen und deren Ergebnisse sich in $\mathrm{Ab}$ hängigkeit von der Struktur der Daten, also der Anzahl der betrachteten Knoten und ihrer Verbindungen, gestalten. Da einige dieser Algorithmen aufgrund ihrer Komplexität extrem rechenintensiv sind, erfordern sie dementsprechend eine leistungsstarke IT-Struktur [27].

Die Anwendung von CommunityDetection-Algorithmen auf die ärztlichen Abrechnungsdaten für die Unterscheidung von PSN kann dazu führen, dass eine modulare Untergliederung in Teilnetzwerke erst ab einer bestimmten Mindestzahl gemeinsam behandelter Patienten resultiert, weil erst dann eine Struktur mit hinreichend hohem Modularitätswert herausgearbeitet wird.

Landon et al. untersuchen erstmals ausführlich Eigenschaften von PSN auf Basis von Medicare-Daten, ohne dass für die Netzbildung hierbei von einem normativen Mittelpunkt (etwa Hausarzt oder Krankenhaus) ausgegangen wird [28]. In einer Vorläuferarbeit von Barnett et al. stehen noch krankenhausbasierte PSN im Vordergrund, die mit netzwerkanalytischen Methoden auf ihre Netzeigenschaften, die Versorgungsintensität und die damit verbunden Ausgabeneffekte untersucht werden [29]. Barnett et al. kommen zu dem Ergebnis, dass Krankenhäuser mit einem ausgeprägten Facharztnetzwerk eine höhere Versorgungsintensität und systematisch höhere Behandlungskosten haben als Krankenhäuser, die Teil eines hausarztbasierten Behandlernetzwerkes sind, und widersprechen damit in Teilen den Befunden von Pollack et al. [18]. Ob und inwieweit widersprüchliche Ergebnisse auch durch die Definitionen und Methoden der Netzwerkbildung beeinflusst sind, ist bisher nicht beantwortet.

In ihrer grundlegenden Arbeit gehen Landon et al. deshalb über bisherige Ansätze hinaus und beschreiben fakti- sche Vernetzungsstrukturen [28]. Diese sind sehr heterogen und können Ärzte mit und ohne Krankenhausaffiliation, unterschiedliche Fachgruppen in unterschiedlichen Anteilsverhältnissen, Netzwerke ohne Krankenhausbeteiligung sowie Netzwerke mit mehreren Krankenhäusern umfassen. Die Analyse zielt darauf ab, die Eigenschaften für ein „organisches“ Wachstum von Vernetzungsprozessen zu identifizieren. Die Autoren finden zahlreiche Belege dafür, dass Netzwerke auf Basis gemeinsamer Eigenschaften von Patienten (Hautfarbe) oder Ärzten (Geschlecht, Krankenhausaffiliation) entstehen und dass Netzwerkstrukturen sich nach Region unterscheiden. Unklar bleibt jedoch, inwieweit diese Prozesse durch das Inanspruchnahmeverhalten von Patienten oder durch das Kooperationsverhalten von Ärzten vorangetrieben werden.

Landon et al. illustrieren exemplarisch die sich in 2 Städten ergebenden Netzwerkstrukturen bei einer Mindestzahl von 10 geteilten Patienten [28]. Dabei resultieren unterschiedliche Netzwerkgrößen (1391 bzw. 596 Ärzte) mit unterschiedlicher Fachgebietsbeteiligung, unterschiedlichen Subnetzwerken und Krankenhausaffiliationen. Diese unterscheiden sich deutlich von den im ersten Schritt normativ gesetzten Netzkonzeptionen mit einem definierten Kristallisationspunkt oder spezifischen zugelassenen Kooperationsbeziehungen zwischen Ärzten ausgewählter Fachrichtungen.

Die Herangehensweise ermöglicht es, Einflüsse von Rahmenbedingungen oder des Verhaltens von Akteuren in der resultierenden Netzwerkstruktur erfassen zu können, ohne dass deren Darstellung durch das normative Setzen von Kristallisationspunkten (z. B. Hausarzt, Krankenhaus) bereits verzerrt oder eingeschränkt ist. Wie Landon et al. gezeigt haben, weisen die mittels graphentheoretisch fundierter Methoden gefundenen Vernetzungsstrukturen eine große Heterogenität auf, sodass bei dieser Herangehensweise aus einer Grundgesamtheit verschiedene Gemeinschaften identifiziert werden, die entweder eine starke Häufung von Hausärzten und/oder Krankenhäusern enthalten oder ganz ohne diese 
bisher üblichen normativ gesetzten Kristallisationspunkte bestehen können (etwa nur Chirurgen und Anästhesisten) [28].

Wie sich die eingesetzten Methoden der Communitydetection auf die Zusammensetzung der resultierenden PSN und diese wiederum auf die dann als "gemeinschaftlich“ betrachteten Behandlungsprozesse und Outcomes auswirken, muss erst noch gezeigt werden. Erkennbar wird, dass die Bildung von Gemeinschaften anhand der geteilten Patienten nicht zwingend mit beabsichtigter Kooperation der Akteure (Ärzte, Krankenhäuser) gleichgesetzt werden kann.

Ein interessantes Beispiel, wie Kooperation beschrieben und erkannt werden kann, geben Carson et al. [30]. Mit Daten einer stationär genutzten elektronischen Patientenakte beschreiben die Autoren ein Modell zur Bewertung von Kooperationsbeziehungen zwischen allen am Behandlungsprozess beteiligten Ärzten und Pflegern („Versorger“). Dabei wurde die Interaktion der Beteiligten jeweils als paarweise Verbindung je geteiltem $\mathrm{Pa}$ tient erfasst und durch Behandlungsprozesse („activities“) und Outcomeparameter (Ergebnisse eines Patient-Satisfaction-Surveys) beschrieben. Zur Bewertung der Interaktionen entwickeln die Autoren das "shared positive outcome ratio“ (SPOR), das die beobachtete Anzahl risikoadjustierter Outcomes für die Interaktionen zweier Versorger im Vergleich zum erwarteten Outcome darstellt. Höhere SPOR-Werte wurden für Versorger gefunden, die eine größere Zahl von Interaktionen und geteilten Patienten aufwiesen [30]. Dies legt aus Sicht der Autoren nahe, dass erfahrenere Versorger auch erfolgreicher kooperieren können. Zugleich zeigt die Studie, dass die meisten Versorger jeweils gleichzeitig in besser und schlechter bewertete Kooperationen eingebunden waren, die Qualität der Verbindungen sich somit in hohem Maße variabel darstellt.

\section{Erste explorative Analysen in Deutschland}

Stillfried und Czihal berichten erstmals von einer Darstellung virtueller Behand- lernetzwerke auf der Grundlage vertragsärztlicher Abrechnungsdaten der Kassenärztlichen Vereinigungen [31]. Die Autoren zielen primär auf die Bildung distinkter Patientenpopulationen ab und wählen dafür den „usual provider“ aus dem hausärztlichen Versorgungsbereich als Kristallisationspunkt der Netzwerkbildung. Alle weiteren Kontakte der Patienten $\mathrm{zu}$ anderen Vertragsärzten tragen zur Bildung der PSN bei, denen dann sämtliche vertragsärztlichen Leistungen und Outcomes der ambulanten Versorgung ihrer Patientenpopulationen zugerechnet werden können. Es zeigen sich ähnliche systematische Einflüsse wie in den USA: Eine höhere Morbidität der Patienten sowie eine städtische Umgebung sind tendenziell mit größeren PSN assoziiert. Die Ausprägungen prozessbezogener Qualitätsindikatoren (z.B. Diabetespatienten mit HbA1c-Messung, Einsatz bildgebender Verfahren bei Patienten mit Rückenschmerzen) weisen hingegen eine wesentlich höhere Varianz zwischen den PSN als räumliche Bezüge auf. Dieser Befund spricht tendenziell gegen die These, dass der Wohnort von Patienten schicksalhaft über deren Versorgungsqualität entscheide [32].

Indikationsspezifische Anwendungen zeigen, dass die Netzwerkanalyse Besonderheiten der Versorgungsstrukturen aufdecken und so Ansatzpunkte für gezielte Nachforschungen und Verbesserungsmaßnahmen liefern kann. Riens und Bätzing-Feigenbaum nutzen hausarztbasierte PSN zur Analyse der ärztlichen Versorgung von Patienten mit Herzinsuffizienz [33]. Zwischen den PSN bestehen erhebliche Unterschiede in der Verordnung der in den Leitlinien empfohlenen Wirkstoffe. Daneben werden systematische Unterschiede in der Behandlung männlicher und weiblicher Patienten (durchgängig geringere Leitlinienadhärenz für weibliche Patienten) sowie regionale Unterschiede (höhere Wahrscheinlichkeit einer leitliniengerechten Verordnung in den neuen Bundesländern) deutlich. Für geschlechtsspezifische Verordnungsunterschiede finden sich plausible Gründe (vgl. [34]). Der Vergleich zwischen den PSN offenbart jedoch erhebliche Niveauunterschiede, die Raum für Ver- besserungen zulassen. Dies reflektiert Ergebnisse von Bynum et al. zur Analyse der Leitlinienadhärenz in der Diabetesbehandlung in den USA [35]. Stillfried et al. untersuchen mit hausarztbasierten PSN regionale Besonderheiten der Demenzversorgung [36]. Dies offenbart, dass die Mehrheit der Demenzpatienten in PSN behandelt wird, die wenige prävalente/inzidente Demenzpatienten versorgen. Die Zahl der Patienten je PSN ist mehrheitlich so gering, dass diese PSN als Gruppe zusammengefasst werden müssen, um systematische Effekte zu erkennen. Eine Minderheit der Demenzpatienten hingegen wird durch PSNversorgt, in denen die hausärztlichen Praxen einen Versorgungsschwerpunkt Demenz aufweisen. Auffällig sind die von der Zahl der Demenzpatienten je PSN und dem Spezialisierungsgrad der Hausarztpraxen abhängigen Unterschiede in Diagnose- und Therapiemustern. Während Patienten in PSN mit geringem Demenzfokus häufiger Antidementiva, aber auch oft Antipsychotika erhielten, wichen PSN mit hohem Demenzfokus systematisch von Leitlinienempfehlungen zur Antidementivaverordnung ab, verordneten aber auch seltener Antipsychotika. Bei gleichem Grundmuster lassen sich zudem regionale Ausprägungen bei Diagnostik und Therapie erkennen.

Koller et al. (2015) und Schang et al. (2017) haben über Vorarbeiten zur PSNAnalyse unter Einbeziehung von Abrechnungsdaten aus der stationären Versorgung referiert [37, 38]. Hierbei werden auf Basis der Daten einer Kassenart erstmals auch indikationsspezifische, krankenhausbasierte PSN für Deutschland ermittelt. Die Autoren berichten z. B. für die Behandlung von Herzinsuffizienzpatienten erhebliche Unterschiede in der Häufigkeit stationärer Aufnahmen zwischen den PSN, die den Befunden von Stukel et al. in Ontario entsprechen [39].

Die vorgenannten Untersuchungen verdeutlichen, welche Möglichkeiten bestehen, um Prozesse in der Versorgung $\mathrm{zu}$ verbessern. Sie werfen die Frage auf, in welchem Umfang die beobachteten Behandlungsmuster in den PSN statisch bzw. veränderlich oder beeinflussbar 
sind. Die bisherigen Vorarbeiten haben daher zu einer erfolgreichen Antragstellung für ein vom Innovationsfonds zu förderndes Versorgungsforschungsprojekt geführt. Das Projekt „Accountable Care in Deutschland" zielt darauf ab, in rund 400 randomisiert ausgewählten PSN beabsichtigte Kooperationsprozesse zur Reduktion vermeidbarer Krankenhausaufenthalte zu stimulieren. Die Eignung von PSN als Plattform, um gezielte Veränderungen von Versorgungsabläufen für eine Patientenpopulation umzusetzen, ist Gegenstand der Evaluation [40].

\section{Werkstattbericht des Zentralinstituts}

Die genannten Arbeiten zu diesem jungen Forschungsgebiet sind noch tastende Versuche, die Komplexität der medizinischen Versorgung zu beschreiben und die aus dieser Transparenz resultierenden Verbesserungspotenziale zu erkennen. Weitergehende Forschungsarbeiten dienen derzeit dazu, die Methoden der Netzwerkanalyse als Grundlage für geeignete Feedbackverfahren an Vertragsärzte weiterzuentwickeln.

\section{Datengrundlage}

Dem Zentralinstitut liegen jeweils vollständig pseudonymisierte vertragsärztliche Abrechnungsdaten sowie Arzneiverordnungsdaten für die rund 70 Mio. gesetzlich versicherten Patienten vor [41]. Die an der vertragsärztlichen Versorgung teilnehmenden Ärzte und Praxen bzw. verordnende Praxen können anhand der Pseudonyme unterschieden werden [42]. Auf dieser Datengrundlage können PSN im Rahmen der ambulanten ärztlichen Tätigkeit für den gesetzlich versicherten Teil der Bevölkerung abgebildet werden. Die vertragsärztlichen Abrechnungsdaten enthalten alle von einer Praxis für einen Patienten abgerechneten vertragsärztlichen Leistungen gemäß der vertragsärztlichen Gebührenordnung (Einheitlicher Bewertungsmaßstab, EBM, vgl. [43]), die Scheinart (Originalschein, Überweisung, Notfall) sowie die quartalsweise dokumentierten behandlungsrelevanten Diagnosen kodiert nach der ICD-10-GM. Die Arzneimittelabrechnungsdaten enthalten je Patient die Pharmazentralnummer der $\mathrm{zu}$ Lasten der gesetzlichen Krankenkassen in einer öffentlichen Apotheke abgegebenen Verordnungen mit Angabe der verordnenden Praxis. Auf Grundlage der in der Betriebsstättennummer enthaltenen 2-stelligen Fachgruppenkennzahl können die Praxen jeweils einer Fachgruppe zugeordnet werden. Vertragsärztliche Abrechnungsdaten und Arzneimittelabrechnungsdaten dürfen im Zentralinstitut für die kassenärztliche Versorgung ( $\mathrm{Zi}$ ) nur für einzelne Studien nach den Voraussetzungen des $\$ 75$ SGB X zusammengeführt werden. Eine Ausnahme bildet die Zusammenführung der Arzneimittelabrechnungsdaten mit den dokumentierten Diagnosen je Patient und verordnender Praxis. Analysen zu geografischen Unterschieden in der Versorgung oder Netzwerkanalysen müssen daher im Zi getrennt für die jeweiligen Datensätze durchgeführt werden. Zur Darstellung der Effekte von PSN auf die Inanspruchnahme von Krankenhausleistungen oder zur Analyse von Behandlernetzwerken zwischen niedergelassenen Ärzten und Krankenhäusern sind Abrechnungsdaten der einzelnen Krankenkassen erforderlich. Eine kassenübergreifende Datengrundlage mit dem erforderlichen Detaillierungsgrad der Datensätze liegt nach unserer Kenntnis nur in der pharmakoepidemiologischen Forschungsdatenbank GePaRD (German Pharmacoepidemiological Research Database) des Leibniz-Instituts für Präventionsforschung und Epidemiologie (BIPS) vor. Diese enthält die Daten von mehr als 14 Mio. Versicherten von 4 gesetzlichen Krankenkassen mit unterschiedlicher regionaler Ausbreitung [44]. Damit liegen in diesen Daten für die meisten Regionen wiederum keine praxisvollständigen Informationen vor, um regionale Besonderheiten der Vernetzungsbeziehungen abbilden $\mathrm{zu}$ können.
Instrumentelle Ansätze hausarztbasierte PSN

\section{Zeitliche Konstanz von Behandlungsmustern in PSN}

In Fortsetzung des bei Stillfried und Czihal beschriebenen Analyseansatzes [31] wurde am Beispiel der HbAlc-Messung bei Diabetikern untersucht, wie sich Indikatoren der Prozessqualität im Zeitablauf innerhalb der Netzwerke verändern. Für das Datenjahr 2010 hatten Stillfried und Czihal rund 43.000 hausarztbasierte Netzwerke gebildet [31]. Zwischen diesen wies der Anteil der Diabetiker mit mindestens einer Langzeitblutzuckermessung im Jahr eine große Variabilität auf, die nicht durch regionstypische Merkmale (städtische/ ländliche Umgebung) beeinflusst wurde. Unklar blieb, ob es sich hierbei um eine Momentaufnahme handelte oder ob der beobachteten Varianz systematische Einflüsse zugrunde lagen. Zunächst wurde die zeitliche Stabilität der PSN überprüft. Dafür wurden für Hausärzte, die über 3 Datenjahre (2012-2014) beobachtet werden konnten, 38.711 hausarztbasierte Netzwerke gebildet, denen nach dem Prinzip des „usual provider“ im hausärztlichen Versorgungsbereich jeweils jahresweise alle Patienten zugeordnet wurden, die jeweils mindestens einen Hausarztkontakt hatten $(N=59.053 .516)$. Rund $4 \%$ aller Patienten wechselten dabei ihr PSN jährlich. Insgesamt ergaben sich im Kernbereich der Verbindungen ( $>10$ geteilte Patienten) kaum Veränderungen hinsichtlich der Größe der PSN. Allerdings stieg die durchschnittliche Anzahl der Ärzte je Netzwerk von 688 (Median 594) in 2012 auf 701 (621) in 2014; erkennbar erfolgte diese Veränderung fast vollständig im Jahr 2013 nach Abschaffung der Praxisgebühr.

Die Ausprägung des Qualitätsindikators wies jedoch deutliche Veränderungen auf. Im Basisjahr lag das arithmetische Mittel der Diabetiker mit mindestens einer HbAlc-Messung für alle Netzwerke bei rund $76 \%$, der Median bei $82 \%$. Zu beobachten war eine 2-gipflige Verteilung der Indikatorausprägung, in der sich zwei unterschiedliche Versorgungsmuster abbilden: In der größeren, leitlinienorientierten Gruppe von PSN 


\begin{tabular}{|c|c|c|c|c|c|c|c|c|}
\hline \multirow{2}{*}{$\begin{array}{l}\text { Netzwerke gemäß } \\
\text { des Anteils der } \\
\text { Diabetiker mit } \\
\text { HbA1c-Test in } 2012\end{array}$} & \multicolumn{4}{|c|}{ Diabetiker mit HbA1c-Test im Jahr 2012} & \multicolumn{4}{|c|}{$\begin{array}{l}\text { Diabetiker mit HbA1c-Test im Jahr } 2014 \\
\text { (Veränderung für gleiche Gruppe von Netzwerken) }\end{array}$} \\
\hline & $\begin{array}{l}\text { Minimalwert } \\
(\%)\end{array}$ & $\begin{array}{l}\text { Maximalwert } \\
(\%)\end{array}$ & $\begin{array}{l}\text { Mittelwert } \\
\text { (\%) }\end{array}$ & $\begin{array}{l}\text { Median } \\
(\%)\end{array}$ & $\begin{array}{l}\text { Minimalwert } \\
(\%)\end{array}$ & $\begin{array}{l}\text { Maximalwert } \\
(\%)\end{array}$ & $\begin{array}{l}\text { Mittelwert } \\
(\%)\end{array}$ & $\begin{array}{l}\text { Median } \\
\text { (\%) }\end{array}$ \\
\hline Bis zu $25 \%$ mit Test & 1 & 25 & 18 & 19 & 1 & 100 & 57 & 68 \\
\hline $25-50 \%$ mit Test & 25 & 50 & 39 & 40 & 6 & 100 & 59 & 56 \\
\hline $50-75 \%$ mit Test & 50 & 75 & 67 & 68 & 4 & 100 & 70 & 72 \\
\hline Mind. $75 \%$ mit Test & 75 & 100 & 87 & 87 & 11 & 100 & 85 & 87 \\
\hline Alle PSN & 1 & 100 & 76 & 82 & 1 & 100 & 79 & 83 \\
\hline
\end{tabular}

Tab. 2 Anteil der Patienten mit mindestens einem Arztbrief im Jahr 2015 für ausgewählte Fachgruppen

\begin{tabular}{|c|c|c|c|}
\hline Fachgruppe & $\begin{array}{l}\text { Anzahl } \\
\text { Ärzte }\end{array}$ & Anzahl Patienten & $\begin{array}{l}\text { Anteil Patienten mit min- } \\
\text { destens einem Arztbrief } \\
\text { (\%) }\end{array}$ \\
\hline Hausärzte & 56.564 & 56.893 .853 & 9,9 \\
\hline $\begin{array}{l}\text { Nichtärztliche Psychothera- } \\
\text { peuten }\end{array}$ & 22.553 & 1.533 .561 & 32,7 \\
\hline Gynäkologie & 12.483 & 18.726 .694 & 11,0 \\
\hline Innere Medizin & 12.248 & 13.986 .796 & 76,3 \\
\hline Kinder- und Jugendmedizin & 7714 & 8.663 .910 & 10,8 \\
\hline Orthopädie & 7212 & 14.769 .765 & 39,4 \\
\hline Chirurgie & 6425 & 9.509 .768 & 45,5 \\
\hline Augenheilkunde & 6246 & 17.655 .139 & 16,5 \\
\hline Ärztliche Psychotherapeuten & 5422 & 479.669 & 26,3 \\
\hline Hals-Nasen-Ohren-Heilkunde & 4411 & 12.462 .550 & 17,7 \\
\hline Radiologie & 4005 & 12.285 .669 & 84,0 \\
\hline Hautärzte & 3945 & 12.915 .198 & 10,9 \\
\hline Anästhesiologie & 3889 & 2.431 .544 & 6,2 \\
\hline
\end{tabular}

lag der Mittelwert für den Anteil der im jeweiligen Netz versorgten Diabetiker mit HbAlc-Messung bei $80 \%$. In der kleineren, leitlinienfernen Gruppe von PSN lag dieser Mittelwert bei $20 \%$ der im Netzwerk versorgten Diabetiker. Über die möglichen Ursachen dieser Gruppenbildung, die von Behandlungsstilen bis zur sozialen Schichtung der jeweiligen Patientenpopulationen reichen können, konnte anhand der verfügbaren Daten keine Aussage gemacht werden. Im Verlauf der 3 Jahre ergaben sich jedoch erhebliche Änderungen.

Wie - Tab. 1 zeigt, haben mehr Netzwerke zur leitlinienorientierten Gruppe aufgeschlossen, nur wenige hingegen haben sich aus der Spitzenreitergruppe in Richtung Leitlinienferne bewegt. So hat sich das arithmetische Mittel aller Diabetiker mit HbAlc-Messung von 76 auf $79 \%$ verbessert, der Median von 82 auf $83 \%$. Während im $25 \%$-Perzentil der Netzwerke mit der geringsten Häufigkeit der HbAlc-Messung der Median von 19 auf $68 \%$ gestiegen ist, blieb er in der Gruppe der am stärksten leitlinienorientiert behandelnden PSN konstant. In dieser Gruppe hatten sich jedoch einige wenige PSN verschlechtert und damit die Spitzengruppe verlassen. In dem PSN, das 2012 noch dem $75 \%$-Perzentil angehörte und 2014 den niedrigsten Anteil der Diabetiker mit HbA1c-Messung aufwies, betrug der Anteil leitliniengerechttherapierter Patienten noch $11 \%$. Auch hier konnten keine Rückschlüsse auf Ursachen gezogen werden. Noch nicht geprüft wurde, ob sich in diesen PSN spezifische Änderungen der Zusammensetzung auf Ärzte- oder Patientenseite ergeben haben. Insgesamt sprechen Richtung und Ausmaß der Veränderlichkeit der Indikatoren dafür, dass die beobachteten Behandlungsmuster keinesfalls schicksalhaft gegeben sind. Offen bleibt, durch welche Maßnahmen gewünschte Veränderungen in den populationsbezogenen Indikatorausprägungen je Netzwerk induziert bzw. gezielt herbeigeführt werden können.

\section{Erkennen ärztlicher Kooperations- beziehungen}

PSN werden im Wesentlichen durch das Patientenverhalten bestimmt. Wenn sich populationsbezogene Qualitätsindikatoren, wie z.B. die Häufigkeit der Diabetespatienten mit einer HbAlc-Bestimmung, im Zeitablauf in den PSN verändern, wirft dies Fragen nach dem Einfluss der Behandler auf die Zusammenarbeit innerhalb von PSN auf. Fraglich ist, wie zwischen den Behandlern gezielt herbeigeführte Kooperationsbeziehungen anhand von Routinedaten erkannt werden können. Grundlage waren hier die im vorangegangenen $\mathrm{Ab}$ schnitt beschriebenen PSN. Aus anderen Studien ist bekannt, dass die Mitbehandlung durch Internisten mit einer höheren Ausprägung prozessualer Qualitätsindikatoren assoziiert ist [45]. Auf Patientenebene konnte diese Assoziation auch in den hier verwendeten Daten beobachtet werden: Unter den Diabetespatienten mit Internistenkontakt war der Anteil mit einer HbAlc-Bestimmung höher. Somit lag die Vermutung nahe, dass der Qualitätsindikator durch die Zahl der gemeinsam von Haus- 


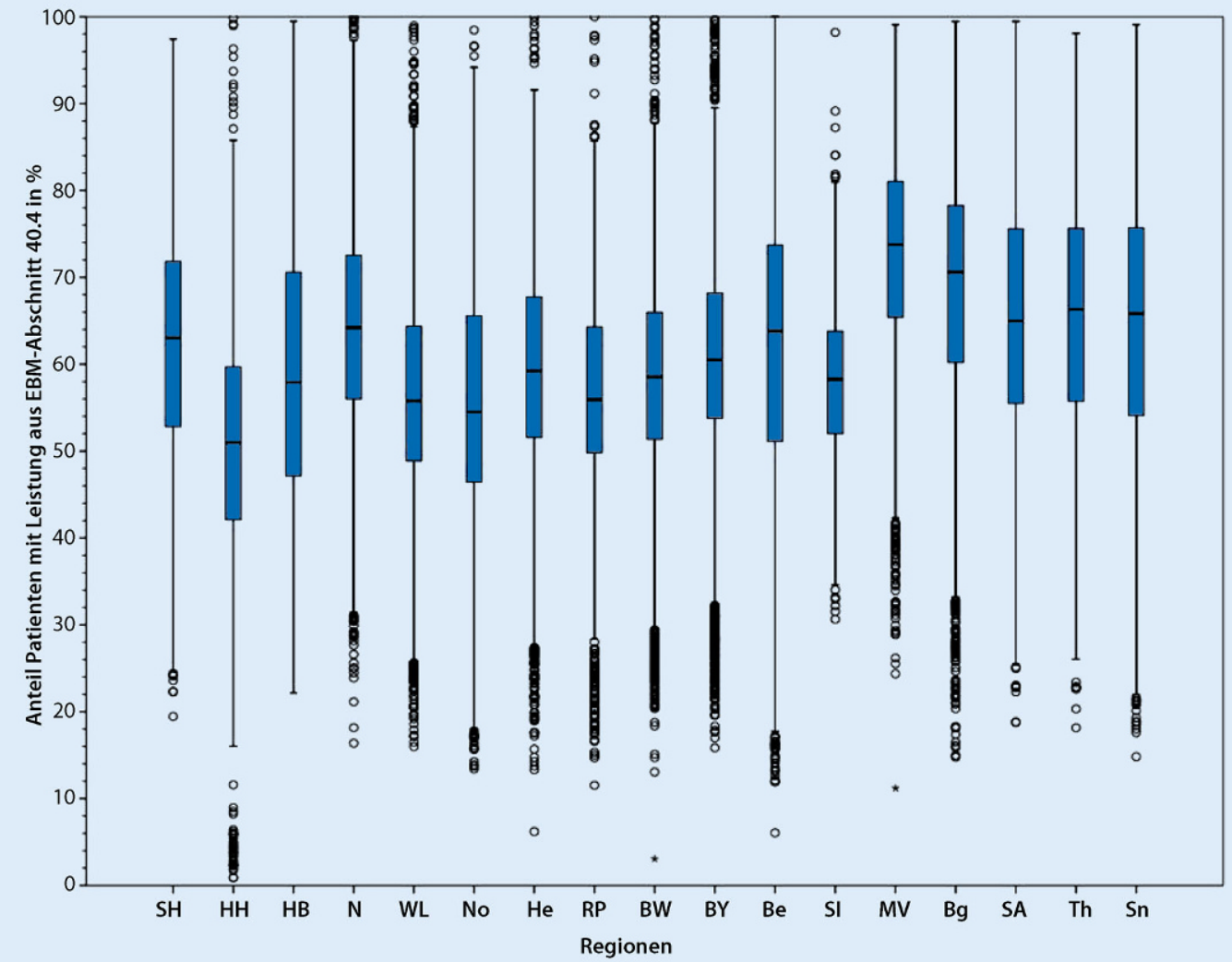

Abb. 1 ॥ Anteil der Patienten mit mindestens einem Arztbrief (Leistung aus Abschn. 40.4 des einheitlichen Bewertungsmaßstabs [EBM]) im Jahr 2015 innerhalb hausarztbasierter "patient-sharing networks" (PSN) nach Regionen. Die Boxplots stellen die Verteilung des Anteils der Patienten mit mindestens einem Arztbrief (Leistung aus Abschn. 40.4 des EBM) je PSN innerhalb der Zuständigkeitsbereiche der 17 Kassenärztlichen Vereinigungen dar. Die räumliche Zuordnung der PSN zu einem Zuständigkeitsbereich einer Kassenärztlichen Vereinigung erfolgte über den Standort der jeweiligen Praxen. SH Schleswig-Holstein, HH Hamburg, HB Bremen, N Niedersachsen, WL Westfalen-Lippe, No Nordrhein, He Hessen, RP Rheinland-Pfalz, BW Baden-Württemberg, BY Bayern, Be Berlin, S/ Saarland, MV Mecklenburg-Vorpommern, Bg Brandenburg, SA Sachsen-Anhalt, Th Thüringen, Sn Sachsen. (Quelle: Eigene Berechnungen auf Basis bundesweiter vertragsärztlicher Abrechnungsdaten für das Jahr 2015)

arzt und Internisten geteilten Patienten bestimmt wird. Dies hätte auch den Befunden von Pollack et al. entsprochen [18]. Gleichwohl konnte - zumindest im Rahmen der hier zugrunde gelegten hausarztbasierten Netzbildung - ein Zusammenhang zwischen dem Qualitätsindikator und der Anzahl gemeinsam von Hausarzt und Internist behandelten Patienten nicht bestätigt werden. Insgesamt waren Verbindungen zwischen Akteuren, die durch eine hohe Anzahl gemeinsamer Patienten charakterisiert waren, hinsichtlich der Ausprägung des Qualitätsindikators den Verbindungen, die nur durch eine geringe Anzahl gemeinsamer Patienten bestimmt waren, nicht überlegen.

Erforderlich sind daher weitere qualifizierende Merkmale zur Identifikati- on von Kooperationsbeziehungen. Nach Rücksprache mit niedergelassenen Ärzten wurde dazu testweise die Abrechnung eines Arztbriefes (Kostenpauschalen für Versendung von Briefen oder Telefax gemäß Abschn. 40.4 des einheitlichen Bewertungsmaßstabs) während des Abrechnungsjahres 2015 herangezogen. $\mathrm{Zu}$ prüfen war die Hypothese, dass die Abrechnung von Arztbriefen als Indikator für eine gezielte Kommunikation oder Kooperation dienen kann. Die Häufigkeit der Abrechnung von Arztbriefen weist große fachgruppenspezifische Unterschiede auf (•Tab.2). Regionale Muster in der Nutzung von Arztbriefen innerhalb hausarztbasierter PSN zeigen sich ebenfalls; die Boxplots in $\mathbf{A b b} \mathbf{1}$ geben die Verteilung des Anteils der Patienten mit mindestens einem Arztbrief innerhalb der PSN der jeweiligen Region an.

Auffällig ist die weit überdurchschnittliche Kommunikation innerhalb der PSN in Mecklenburg-Vorpommern und die eher gering ausgeprägte Nutzung in Hamburg. Im Mittel betrug die Abrechnungshäufigkeit von Arztbriefen je Patient 1,94, für Patienten mit mindestens einem Arztbrief jedoch 3,47. Dies spricht dafür, dass Verbindungen zwischen Akteuren, die Arztbriefe schreiben, von intensiver und auch gegenseitiger Kommunikation geprägt sind. Auf Ebene der PSN wurde ein schwacher, aber positiver Zusammenhang zwischen dem Anteil der Patienten mit Arztbriefen je PSN und dem Qualitätsindikator „Anteil der Diabetiker mit mindestens einer HbAlc-Messung 

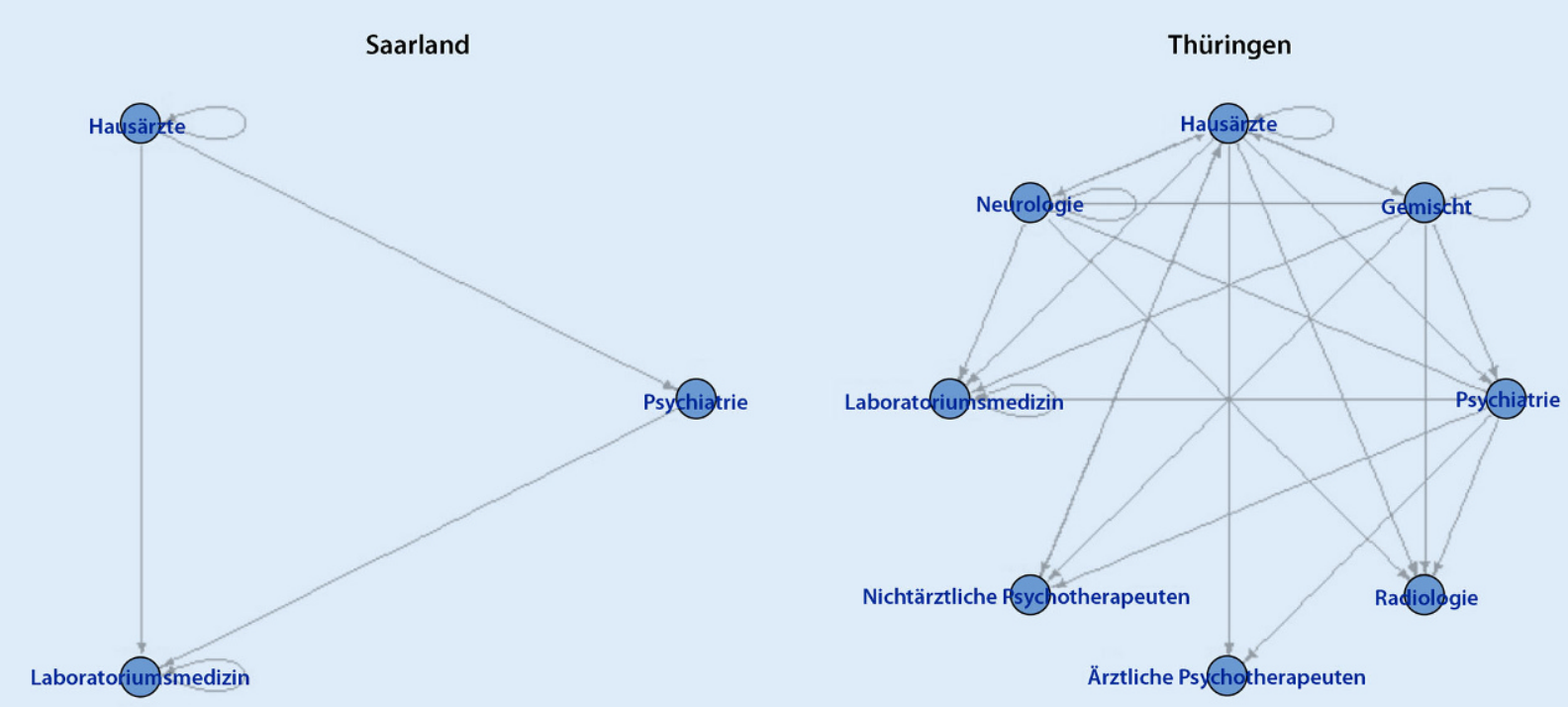

Knotenanzahl: 3

Kantenanzahl: 5

Kantendichte: 0,833

Knotenanzahl: 8

Kantenanzahl: 27

Kantendichte: 0,482

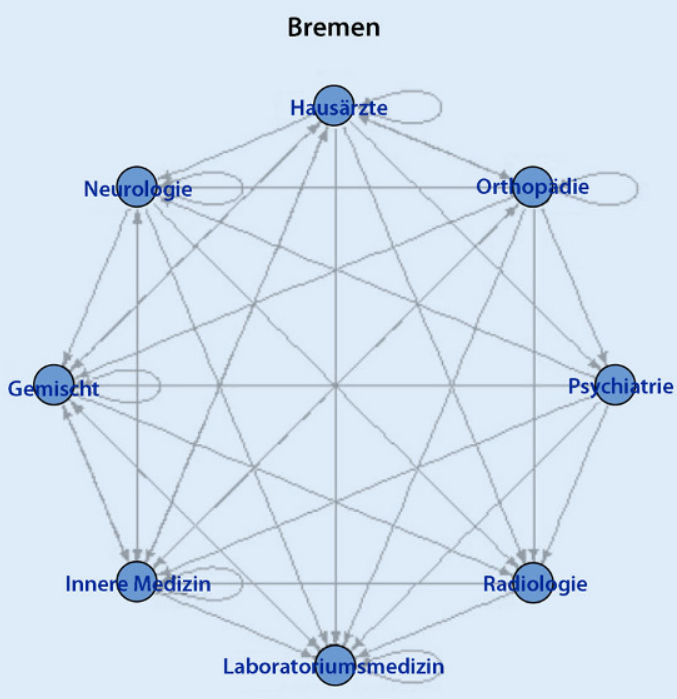

Knotenanzahl: 8

Kantenanzahl: 42

Kantendichte: 0,75

\section{Nordrhein}

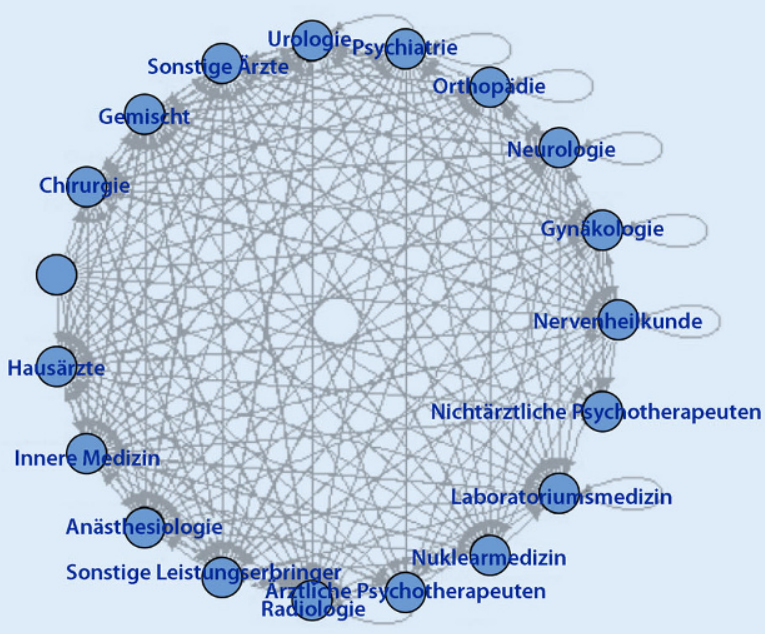

Knotenanzahl: 19

Kantenanzahl: 253

Kantendichte: 0,74

Abb. 2 ム Regionale Unterschiede im Vernetzungspotenzial niedergelassener Ärzte. Die Abbildung zeigt die Anzahl der Verbindungen zwischen allen Praxen der jeweiligen Fachgruppen im Zuständigkeitsbereich ausgewählter Kassenärztlicher Vereinigungen, die durch die Überweisung von Patienten entstehen. Die Fachgruppen werden aggregiert als Knoten betrachtet, die Verbindungen als Kanten, die Dichte der Verbindungen („edge density“) wird grafisch und als Wert ausgewiesen. Grundlage waren Patienten mit einer Überweisung zu/von einem Psychiater. Im Saarland erhalten Psychiater nur Überweisungen von Hausärzten; sie überweisen nur zu Laborärzten. Für einen Teil der Patienten veranlassten auch Hausärzte Laborleistungen oder überwiesen zu anderen Hausärzten. Die größere Zahl von beteiligten Fachgruppen in anderen Regionen erklärt sich zum Teil auch durch die Komorbidität der Patienten. Auf Ebene einzelner "patient-sharing networks" (PSN) können die Beziehungen abweichen. (Quelle: Eigene Berechnungen auf Basis bundesweiter vertragsärztlicher Abrechnungsdaten) 
Tab. 3 Nutzung von Community-Detection-Algorithmen zur Abbildung von „patient-sharing networks" (PSN): exemplarische Darstellung von PSN in 2 Städten bei einem Modularitätswert $>0,3$ Stadt in

Neue Bundesländer Alte Bundesländer

\begin{tabular}{|l|l|l|}
\hline Anzahl betrachteter Patienten & 82.314 & 78.988 \\
\hline Anzahl betrachteter Ärzte & 201 & 163 \\
\hline Anzahl gebildeter PSN & 20 & 22 \\
\hline Anteil Patienten mit Kontakt zu max. einer PSN & $26,4 \%$ & $29,6 \%$ \\
\hline $\begin{array}{l}\text { Anteil Patienten mit Kontakt zu mind. 4 PSN } \\
\text { Quelle: Eigene Berechnungen auf Basis bundesweiter vertragsärztlicher Abrechnungsdaten für das } \\
\text { Jahr 2015 }\end{array}$ & $18,8 \%$ & $20,1 \%$ \\
\hline
\end{tabular}

jährlich“ gefunden (39.679 Netze mit $>200$ Patienten: Korrelationskoeffizient $\mathrm{r}^{2}=0,367^{* *}, p=0,01 ; 28.157$ Netze mit $>1000$ Patienten: $\mathrm{r}^{2}=0,429^{* *}, p=$ $0,01)$. Der Zusammenhang ist in sehr kleinen PSN weniger stark ausgeprägt, in denen - gemäß dem Algorithmus der Netzbildung - der hausärztliche Kristallisationspunkt nur einen geringen Tätigkeitsumfang aufweist. Dieser Befund könnte die Hypothese unterstützen, dass die Abrechnung von Arztbriefen ein qualifizierendes Merkmal der Verbindungen zwischen Akteuren innerhalb von PSN ist.

Analytische Ansätze - Anwendung graphentheoretischer Methoden

\section{Geografische Unterschiede im Kooperationspotenzial}

Wie oben beschrieben kann die gemeinsame Behandlung von Patienten auf der Grundlage von Überweisungen ein Indikator für eine intensivere Kooperation sein. In Regionen, in denen ein höherer Anteil von Patienten auf Grundlage von Überweisungen behandelt wird, wäre demnach generell eine höhere Kooperationsbereitschaft zwischen den Ärzten anzunehmen (Kooperationspotenzial). Wie Landon et al. für die Versorgung von Medicare-Patienten dargelegt haben, weisen PSN, die mit graphentheoretisch fundierten Verfahren ermittelt wurden, deutliche regionale Strukturunterschiede auf [28]. Für Deutschland konnte mit bundesweiten Abrechnungsdaten noch keine vollständige Abbildung der Netzwerkstrukturen mit entsprechenden Verfahren auf Arztebene erstellt werden. Näherungsweise wurde das regionale Vernetzungs- und Kooperationspoten- zial auf Fachgruppenebene analysiert. Datengrundlage waren die bundesweiten vertragsärztlichen Abrechnungsdaten für das 4. Quartal 2015. Berücksichtigt wurden jedoch nur Patienten mit Überweisungen und für diese jeweils der ausstellende Arzt (von wem kommt der Patient?) und der weiterbehandelnde Arzt (zu wem geht der Patient?). Diese Daten wurden auf der Ebene der jeweiligen Arztfachgruppen nach Zuständigkeitsbereichen der Kassenärztlichen Vereinigungen (KV-Region) aggregiert. Jede Fachgruppe wurde somit zu einem „Knoten“, die Patienten mit Überweisungen $\mathrm{zu}$ „Kanten“. In einem weiteren Schritt wurden die Kantendichte sowie andere Netzmerkmale berechnet, die hier jedoch nicht in Gänze dargestellt werden können. Geografische Unterschiede im Kooperationspotenzial sind ausgeprägt. Sie werden hier exemplarisch in $-A b b .2$ veranschaulicht, welche die Unterschiede in der vernetzten Behandlung für $4 \mathrm{KV}$-Regionen zeigt. Auswahlkriterium waren Patienten mit einer Überweisung von oder zu einem Psychiater, wobei die Gesamtheit aller durch Überweisungen für diese Patienten verbundenen Fachgruppen abgebildet wird, auch wenn diese andere, nichtpsychiatrische Behandlungsanlässe betreffen. Aus Datenschutzgründen nicht dargestellt sind Einzelfälle bzw. Verbindungen zwischen Fachgruppen mit einer Anzahl $<30$. Die Berechnungen und Visualisierungen erfolgten in R, Version 3.3.3 ( $\mathrm{R}$ Foundation for Statistical Computing, Wien, Österreich). Im Saarland etwa erhalten Psychiater Überweisungen nur von Hausärzten, sie selbst überweisen nur an Laborärzte; allerdings wurden für diese Patienten auch Laborunter- suchungen von Hausärzten veranlasst. Zudem erfolgten Überweisungen zwischen verschiedenen Hausarztpraxen. In den anderen Regionen, insbesondere in Nordrhein, ergibt sich ein wesentlich komplexeres Bild der Überweisungen $\mathrm{zu}$ und von Psychiatern, zu anderen Fachgruppen und für diese Patienten (auch für die „Komorbidität" dieser Patienten) zwischen anderen Fachgruppen. Im Saarland besteht somit ein sehr eng definiertes Kooperationspotenzial für psychiatrische Patienten (Hausärzte, Psychiater, Laborärzte). Andere Regionen, am ausgeprägtesten Nordrhein, zeigen ein deutlich erweitertes Kooperationspotenzial. In der Liste potenzieller Netzwerkpartner nordrheinischer Psychiater finden sich somit auch Internisten, Orthopäden, Neurologen, Gynäkologen, Urologen, Chirurgen, Anästhesiologen, Radiologen, Nuklearmediziner und Psychotherapeuten. Ausgewiesen ist die Kantendichte („,edge density") als Kennzahl für die Relation der Anzahl faktischer Verbindungen zur Anzahl möglicher Verbindungen zwischen den Knoten. Da auch Überweisungsbeziehungen zwischen Ärzten einer Fachgruppe möglich sind, zählen diese "In-sich-Kanten“ mit (Saarland: 5/6). In weitergehenden Analysen ist zu klären, welche spezifischen Faktoren den beobachteten Kooperationspotenzialen zugrunde liegen. Da hier eine aggregierte Betrachtung vorliegt, können auf der Ebene der einzelnen PSN in der Region erhebliche Unterschiede in der Breite (beteiligte Praxen aus unterschiedlichen Fachgruppen) und Tiefe (Anteil der Patienten mit Überweisung) der Kooperation bestehen. Insofern weisen die regionalen Unterschiede auf aggregierter Ebene auf das jeweilige Vernetzungsund Kooperationspotenzial hin.

\section{Auffinden kohärenter Gemein- schaften mittels Community- Detection-Algorithmus}

Analysen von sozialen Netzwerken zeigen, dass Menschen sich naturgemäß zu Gruppen formieren. Die Menschen einer Gruppe haben meist einen intensiveren Austausch untereinander als mit Menschen einer anderen Gruppe. Verfahren der Communitydetection, 


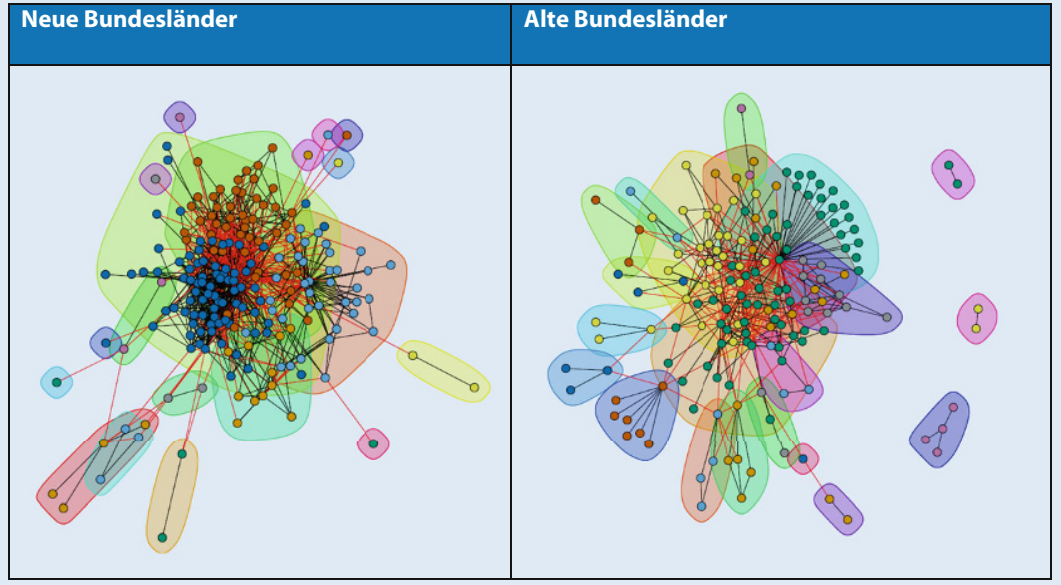

Abb. 3 A Nutzung von Community-Detection-Algorithmen zur Abbildung von „patient-sharing networks" (PSN): exemplarische Darstellung von PSN in 2 Städten bei einem Modularitätswert $>0,3$. Für die Praxen mit Standort in zwei vergleichbar großen Städten (Einwohnerzahl ca. 100.000) wurden die Verbindungen zwischen Ärzten (Knoten bzw. Punkte) aufgrund von überwiesenen Patienten (Kanten bzw. Verbindungen zwischen den Knoten) mit Wohnort in der jeweiligen Stadt ermittelt und mithilfe des Walktrap-Algorithmus aus dem resultierenden Gesamtnetzwerk (farbliche Umrandung) einzelne Gemeinschaften (PSN) identifiziert. Die Abbildung zeigt die grafische Darstellung der resultierenden Gemeinschaften und ihrer Verbindungen untereinander. Kennzahlen hierzu liefert $\mathbf{0}$ Tab. 3. (Quelle: Eigene Berechnungen auf Basis bundesweiter vertragsärztlicher Abrechnungsdaten für das Jahr 2015)

wie sie etwa in der Social-Media-Analyse eingesetzt werden, versuchen die Akteure zusammenzufassen, die untereinander möglichst viele und mit anderen Akteuren möglichst wenig Informationen austauschen [46]. Konzepte des Communitybuilding [26] wurden im $\mathrm{Zi}$ erstmalig auf die vertragsärztlichen Abrechnungsdaten des Gesamtjahres 2015 übertragen, um PSN zu identifizieren. Zur Veranschaulichung werden Ergebnisse für je eine Stadt in den alten und neuen Bundesländern dargestellt. In der Darstellung berücksichtigt wurden nur Patienten mit Wohnort in der jeweiligen Stadt, die von Ärzten am Standort eine Überweisung erhalten oder diese mit einer Überweisung aufgesucht hatten. Ärzte (Knoten) werden über gemeinsame Patienten (Kanten) miteinander verbunden. Mithilfe des Walktrap-Algorithmus [47] werden aus der Vielzahl aller Verbindungen PSN identifiziert. Der Walktrap-Algorithmus ist eines von vielen möglichen Verfahren, um eine Communitystruktur zu erkennen. Der Algorithmus basiert auf dem Prinzip, dass der Lauf bei einer zufälligen „Traversierung“ des Netzwerkes im Beziehungsnetz einer Community "gefangen“ wird, die weniger Verbindungen nach außen als nach innen hat. Die Abgegrenztheit der hierdurch identifizierten PSN wird anhand des Modularitätswertes bestimmt. Sind die internen Verbindungen innerhalb der PSN zahlreich und die Verbindungen zwischen den PSN gering, ist die Modularität hoch. Ein Modularitätswert von über 0,3 kann als Indikator für eine signifikante Communitystruktur gelten [48]. Für die hier dargestellten Beispiele wurden Modularitätswerte von $>0,3$ erreicht, nachdem die Anzahl geteilter Patienten auf $\geq 15$ (alte Bundesländer) und $\geq 25$ (neue Bundesländer) als Bedingung für eine kohärente Verbindung zwischen zwei Ärzten festgelegt wurde. Ein Weglassen dieser Bedingung hätte zur Folge, dass im Netzwerk zu viele Verbindungen existieren und somit keine oder lediglich eine Communitystruktur mit einem relativ niedrigen Modularitätswert identifiziert würde.

Die zentralen Ergebnisse sind in - Tab. 3 dargestellt; - Abb. 3 liefert die grafische Veranschaulichung. In der Stadt der neuen Bundesländer werden trotz größerer Arztzahl weniger Communities gefunden; die Grafik zeigt eine starke
Verdichtung auf wenige große Communities mit „Satelliten“. In der Stadt der alten Bundesländer sind die Communities eher kleiner. Die PSN jeder Stadt unterscheiden sich stark in ihrer Fachgruppenzusammensetzung (hier nicht weiter dargestellt), PSN ohne Hausärzte etwa stehen PSN mit einer größeren Anzahl von Hausärzten gegenüber. Im Gegensatz zum „normativen“ Ansatz der hausarztbasierten PSN werden durch das Community-Detection-Verfahren auch kaum distinkte Patientenpopulationen gebildet. Nur ca. 25-30\% aller Patienten finden sich in einer distinkten Population (Kontakt zu maximal einem PSN), rund $20 \%$ aller Patienten haben zu 4 und mehr Ärztecommunities Kontakt. Die weniger konzentrierte Communitystruktur in der Stadt der alten Bundesländer scheint mit einer etwas höheren Patientenbindung einherzugehen. Um die Bindungskraft bzw. Verantwortung der Ärztecommunities besser einschätzen zu können, müssten zukünftig die Verbindungen, z. B. mit Anzahl oder Wert der erbrachten Leistungen, bewertet werden.

\section{Diskussion}

Die hier vorgestellten Analysen zeigen, dass es grundsätzlich möglich ist, regionale Unterschiede in der Versorgung auf das Handeln einzelner Personen, Patienten und Behandler zurückzuführen. Sofern die erforderlichen Daten vorliegen, ergibt sich hieraus ein Gestaltungsauftrag, die an der Versorgung Beteiligten in die Lage zu versetzen, unerwünschte Prozesse oder Outcomes nach Möglichkeit zu vermeiden. In Gesundheitssystemen mit einem strengen Gatekeepersystem und regionaler Zuständigkeit sind die methodischen Anforderungen hierzu geringer als in Versicherungssystemen, in denen die Versicherten nicht auf die Inanspruchnahme jeweils eines regional zugeordneten Primärversorgers verpflichtet sind. Dort entstehen durch freie Arztwahl und die hieraus resultierende gemeinsame Behandlung von Patienten außerordentlich komplexe Beziehungen zwischen den an der Versorgung beteiligten Einrichtungen. Die Frage, welche Bedeutung diese Beziehungen für die Versorgungsqualität im Alltag haben, wird 
bisher vorwiegend unter dem Gesichtspunkt der Koordination der Versorgung untersucht; wenig überraschend wird die Bedeutung einer guten Abstimmung bzw. eines ärztlichen Koordinators insbesondere bei chronisch kranken Patienten empirisch belegt [49-51]. Hierbei bleibt allerdings offen, welche Faktoren eine gute Koordination der Versorgung bewirken.

Die Analyse der Eigenschaften, Kooperationsbeziehungen und Outcomes der sich selbst organisierenden virtuellen Behandlernetzwerke, hier als PSN bezeichnet, hat unseres Erachtens das Potenzial, diese Lücke zu schließen und die Analyse räumlicher Versorgungsunterschiede sinnvoll zu ergänzen. Hierbei stehen vor allem zwei Fragen im Vordergrund: 1. Inwieweit sind geografische Unterschiede in der Versorgung auf Eigenschaften der geografischen Räume zurückzuführen? 2. Wie können die in bestimmten geografischen Regionen gefundenen unerwünschten Besonderheiten der Versorgung verändert werden? Wie auch bei der Analyse geografischer Besonderheiten sind hierbei Abrechnungsdaten am besten geeignet, ein möglichst vollständiges Bild der Versorgung im Alltag zu zeichnen. Notwendig ist jedoch das Vorliegen eines (pseudonymisierten) Versichertenund Leistungserbringerbezugs, damit das durch parallele oder sequenzielle Behandlungen derselben Patienten entstehende Beziehungsgeflecht zwischen Versorgungseinrichtungen dargestellt werden kann.

Die bisherigen Arbeiten zur Darstellung und Analyse der PSN versprechen, dass durch diese Herangehensweise auf beide Fragen Antworten gefunden werden können. So weisen strukturelle Unterschiede zwischen PSN in unterschiedlichen Regionen auf die Bedeutung von Rahmenbedingungen hin. Wichtiger ist, dass durch die Identifikation von PSN potenziell konkrete Ansprechpartner erkennbar werden, um in einzelnen Regionen konkrete Maßnahmen zur Reduzierung unerwünschter Besonderheiten bzw. zur Verbesserung der Versorgung $\mathrm{zu}$ implementieren.

Erste Ansätze in diese Richtung in den USA und Kanada zielten daher darauf, Informationsgrundlagen für die Bildung von Accountable-Care-Organisationen zu schaffen, indem den infrage kommenden Versorgern Transparenz über ihre Bedeutung als "usual provider" für eine bestimmte Patientenpopulation vermittelt wird. Damit soll einerseits die Bildung neuer Organisationsstrukturen und andererseits die Darstellung der gemeinsam erzielten Versorgungsqualität unterstützt werden. Für Letzteres ist die Abbildung einer möglichst distinkten Patientenpopulation erforderlich, deren Versorgung primär im Verantwortungsbereich des jeweiligen PSN liegt. Diese Ansätze basieren insofern auf einer normativen Auswahl von Vernetzungszentren, zumeist ein Krankenhaus oder ein Hausarzt, denen nach dem Prinzip des "usual provider" Patienten und die von diesen Patienten ebenfalls in Anspruch genommenen Versorgungseinrichtungen zugeordnet werden. Damit werden die faktisch erfolgten Inanspruchnahmeund Kooperationsbeziehungen nach dem Muster eines fiktiven Primärarztsystems geordnet.

Die Stärke dieses Verfahrens besteht insbesondere darin, dass die versicherte Population nicht mehr nach dem Wohnort, sondern nach Art ihrer Inanspruchnahme bzw. Versorgung in distinkte Subpopulationen aufgeteilt werden kann. Allerdings können sich bestimmte Versorgungseinrichtungen dann als Mitglied in mehr als einem PSN wiederfinden. Eine weitere Schwäche offenbart sich im Vergleich zu PSN, die mittels Community-Detection-Algorithmen auf Basis der graphentheoretischen Modelle gebildet werden. Diese PSN sind sowohl in Größe und $\mathrm{Zu}-$ sammensetzung wesentlich heterogener als die nach dem Prinzip des „usual provider" normativ gebildeten PSN. So finden sich z. B. große Netzwerke mit vielen Hausarztpraxen und Krankenhäusern, andere Netzwerke gänzlich ohne diese. Dies spricht dafür, dass Ansätze, die von definierten Kristallisationspunkten (z. B. Krankenhaus, Hausarzt) oder als relevant definierten Kooperationspartnern (z. B. Hausarzt und Internist) ausgehen, viele Inanspruchnahme- und Kooperationsprozesse ausblenden, die im Versorgungsalltag Bedeutung haben können.
Sowohl die normativen als auch die graphentheoretisch fundierten Methoden der Identifikation von PSN haben ihre Limitationen. Keines der Verfahren liefert letztlich „wahre“ Gemeinschaften. Die Anwender graphentheoretisch fundierter Verfahren dürften sich der enormen Komplexität der Aufgabe aber stärker bewusst sein. Denn die Identifikation der Zusammengehörigkeit einer bestimmten Gemeinschaft kann aus der Fülle der in der Versorgungsrealität faktisch vorliegenden Beziehungen letztlich nicht mit Sicherheit, sondern nur durch Vergleich mit einer angenommenen zufälligen Verteilung der Beziehungen bestimmt werden. Die PSN sollten insofern nur als Instrumentalvariablen, z. B. für weitergehende Analysen oder einen Dialog mit den Beteiligten über Art und Umfang ihrer Kooperation, genutzt werden. Die Schwächen der graphentheoretisch fundierten Verfahren bestehen darin, dass unter Nutzung, z. B. der vollständigen Abrechnungsdaten einer KV-Region, teilweise sehr große PSN von mehreren hundert Praxen resultieren. Um eine größere Trennschärfe zu erreichen, muss die Zahl der mindestens gemeinsam behandelten Patienten erhöht werden. Damit werden bestimmte Teile des versorgten Patientenkollektivs ausgeblendet. Zudem sind die Patientenkollektive der mit Community-Detection-Algorithmen gefundenen PSN nicht überschneidungsfrei. Versorgungsergebnisse eines PSN können somit nicht eindeutig dem Verhalten von Patienten oder der Versorger eines bestimmten PSN zugerechnet werden.

Wenn die Arztpraxen bzw. Versorgungseinrichtungen als Akteure definiert werden, liegt es in der Methode der graphentheoretisch fundierten Verfahren, die PSN im Hinblick auf die Leistungserbringer tendenziell überschneidungsfrei abzuleiten. Uns ist bisher keine Anwendung bekannt, bei der die Patienten als Akteure definiert werden, um ein umgekehrtes Bild („doctor-sharing network") $\mathrm{zu}$ erzeugen. Denkbar wäre, dass dann in einem weiteren Schritt beide Abbildungen zusammengeführt werden, um möglichst distinkte Praxisgemeinschaften mit möglichst distinkten Patientenpopulationen zu identifizieren. 
Trotz ihrer Limitationen können Netzwerkanalysen dazu beitragen, räumliche Besonderheiten von nichträumlichen systematischen Effekten (z. B. geschlechtsspezifische Besonderheiten in der Versorgung) und individuellem Verhalten abzugrenzen. Eine besonders große oder geringe Varianz der Prozessoder Outcomeparameter (z. B. Häufigkeit empfohlener Untersuchungen, Häufigkeit von Krankenhausaufnahmen) zwischen den PSN einer Region stellt bereits eine regionale Besonderheit dar und liefert Hinweise darauf, welche Versorgergemeinschaften und ggf. welche Patientenpopulationen näher untersucht, welche Mitglieder bestimmter PSN informiert und ggf. für Maßnahmen eines populationsbezogenen Qualitätsmanagements gewonnen werden sollten. Damit könnten netzwerkanalytische Verfahren dazu beitragen, aus regionalen Versorgungsunterschieden wirksame Gestaltungsaufträge zur Weiterentwicklung von Versorgungsstrukturen und -prozessen abzuleiten.

Die zeitliche Variabilität von Prozessparametern innerhalb der PSN, die am Beispiel der HbAlc-Messung bei Diabetespatienten in der vertragsärztlichen Versorgung dargestellt wurde, spricht für eine grundsätzliche Beeinflussbarkeit der populationsbezogenen Mittelwerte durch die PSN; das Potenzial für eine gezielte Veränderung, z.B. durch Anstreben eines Zielwerts für die gemeinsam behandelte Patientenpopulation, muss jedoch je nach Sachzusammenhang bestimmt werden. Aufgrund der bekannten Substitutionseffekte in der medizinischen Arbeitsteilung zwischen Ärzten unterschiedlicher Fachgruppen, ärztlichen Leistungen und verordneten bzw. veranlassten Leistungen sowie zwischen der ambulanten und der stationären Versorgung [52-54] sind hierfür unbedingt patientenvollständige, sektorenübergreifende Datengrundlagen wünschenswert, um Fehlschlüsse zu vermeiden.

Hierbei ermöglichen beide Verfahren, der normative und der graphentheoretisch fundierte Ansatz, den in der Versorgung aktiven Ärzten einen Überblick über ihre Stellung im Versorgungssystem aus der „Vogelperspektive“ zu geben. An- zunehmen wäre, dass die graphentheoretisch hergeleiteten PSN eher der individuellen Wahrnehmung der Betroffenen entsprechen. Bisher liegen jedoch noch keine vergleichenden Analysen dazu vor, wie ein entsprechendes Feedback von Ärzten und Krankenhäusern im Alltag aufgenommen wird. Ebenso wenig ist bekannt, ob und wie die Versorger in den PSN die Zurechnung von Versorgungsergebnissen akzeptieren oder wie sie die entsprechenden Informationen für abgestimmte Interventionen nutzen können.

Spätestens zu dem Zeitpunkt, an dem eine Intervention zur Verbesserung von Versorgungsabläufen angestrebt wird, wird es notwendig sein, besser zwischen beabsichtigten Kooperationsbeziehungen und unbeabsichtigtem „patient-sharing " und ihren jeweiligen Versorgungsergebnissen zu unterscheiden. Überweisungen, Einweisungen und Arztbriefe können mögliche Hinweise auf beabsichtigte Kooperationsbeziehungen sein, deren Bedeutung für populationsbezogene Outcomes noch weiter erhärtet und die durch weitere Indikatoren einer intendierten und erfolgreichen Zusammenarbeit ergänzt werden sollten. Anhand der Routinedaten für die vertragsärztliche Versorgung in Deutschland haben wird dargelegt, dass die Beziehungsgeflechte, die allein auf Basis von Überweisungen zustande kommen bzw. unter Nutzung von Arztbriefen zur gegenseitigen Kommunikation entstehen, strukturelle Unterschiede sowohl in geografischer Hinsicht als auch zwischen den PSN aufweisen. Anhand der Routinedaten lassen sich erste Hinweise für einen $\mathrm{Zu}$ sammenhang zwischen einem höheren Anteil von Patienten mit Arztbriefen und Ausprägungen von Indikatoren der Prozessqualität je PSN finden, wobei dies bisher nur für hausarztbasierte PSN (ohne Anwendung graphentheoretisch fundierter Verfahren) gezeigt wurde. Entsprechende strukturelle Unterschiede können in weiteren Analysen genutzt werden, um auf Effekte beabsichtigter Kooperationen im Hinblick auf Prozessund Ergebnisqualität zu schließen. Hierbei ist zu berücksichtigen, dass es - wie die Studie von Carson et al. zeigt [30] auch im Rahmen beabsichtigter Kooperationsbeziehungen je nach Akteuren zu
Unterschieden in Prozess- und Ergebnisqualität in der Versorgung kommen kann.

Bei der Abbildung von Qualitätsdimensionen zeigen sich allerdings schnell Grenzen der Abrechnungsdaten. Da diese Daten insbesondere bei pauschalierten Vergütungssystemen stark aggregiert sind und keine Befunddaten enthalten, kann ein erheblicher Teil der Qualitätsindikatoren mit abrechnungsbezogenen Routinedaten nicht abgebildet werden. Zudem bestehen Einschränkungen der Validität für die Qualitätsmessung, die bei einer Verwendung von Routinedaten als Grundlage für ein Feedback zur Verbesserung der Versorgungsqualität berücksichtigt werden müssen [55]. Das Feedback anhand von Routinedaten ist daher nur als ein erster Schritt in einem Dialog mit Praxen und Krankenhäusern zu sehen, der umgekehrt den beteiligten Versorgungsforschern wertvolle Hinweise zum besseren Verständnis ihrer Daten geben wird.

Es wird erwartet, dass einige der hier aufgeworfenen methodischen Fragen zur Identifikation von PSN sowie zur instrumentellen Bedeutung von PSN für Interventionen im Versorgungsalltag im Rahmen des bereits zitierten, vom Innovationsfonds geförderten Projekts „Accountable Care in Deutschland“ beantwortet werden können.

\section{Korrespondenzadresse}

\section{Dr. D. von Stillfried}

Zentralinstitut für die kassenärztliche Versorgung

Salzufer 8, 10587 Berlin, Deutschland dstillfried@zi.de

\section{Einhaltung ethischer Richtlinien}

Interessenkonflikt. D. von Stillfried, T. Ermakova, F. Ng und T. Czihal sind Angestellte des Zentralinstituts für die kassenärztliche Versorgung in Deutschland (Zi). Das Zi ist eine Stiftung des bürgerlichen Rechts, die von den 17 Kassenärztlichen Vereinigungen (KVen) finanziert wird. Satzungsgemäß unterstützt die Forschung des Zi die KVen bei der Wahrnehmung ihres gesetzlichen Sicherstellungsauftrags. Der Erstautor ist in der International Wennberg Collaboration engagiert, einer Verbindung von Wissenschaftlern, die aus der Erforschung regionaler Unterschiede Ansätze zur Gestaltung der medizinischen Versorgung ableitet. Das Zi erhält Gelder aus dem Innovationsfonds als Teil des 
Projektkonsortiums „Accountable Care in Deutschland (ACD)", das mit den beschriebenen Methoden arbeitet. Bei keinem der Autoren besteht ein Interessenkonflikt durch anderweitige Beraterhonorare oder Beschäftigungsverhältnisse.

Dieser Beitrag beinhaltet keine von den Autoren durchgeführten Studien an Menschen oder Tieren. Für die aufgeführten Studien gelten die jeweils dort angegebenen ethischen Richtlinien.

Open Access. Dieser Artikel wird unter der Creative Commons Namensnennung 4.0 International Lizenz (http://creativecommons.org/licenses/by/4.0/deed. de) veröffentlicht, welche die Nutzung, Vervielfältigung, Bearbeitung, Verbreitung und Wiedergabe in jeglichem Medium und Format erlaubt, sofern Sie den/die ursprünglichen Autor(en) und die Quelle ordnungsgemäßnennen, einen Linkzur Creative Commons Lizenz beifügen und angeben, ob Änderungen vorgenommen wurden.

\section{Literatur}

1. Wennberg J, Gittelsohn A (1973) Small Area Variations in Health Care Delivery, Science 182(4117):1102-1108

2. OECD (2014) Geographic variations in health care: what do we know and what can be done to improve health system performance? OECD Health Policy Studies. OECD Publishing, Paris

3. Czihal T, von Stillfried D, Schallock M (2012) Mitversorgungsbeziehungen in der ambulanten Versorgung (Teil 1). Mitversorgung für andere Regionen (2008). Versorgungsatlas-Bericht, Bd. $12 / 04$. Zentralinstitut für die kassenärztliche Versorgung in Deutschland (Zi), Berlin https:// doi.org/10.20364/VA-12.04 (Zugegriffen: 31. Juli 2017)

4. The Trustees of Dartmouth College (2017) The Dartmouth atlas of health care. http://www. dartmouthatlas.org/data/region/. Zugegriffen: 31. Juli 2017

5. Health Quality \& Safety Commision New Zealand (2017) https://www.hqsc.govt.nz/ our-programmes/health-quality-evaluation/ projects/atlas-of-healthcare-variation/gout/. Zugegriffen:31. Juli 2017

6. Barker I, Steventon A, Deeny SR (2017) Association between continuity of care in general practice and hospital admissions for ambulatory care sensitive conditions: cross sectional study of routinely collected, person level data. BMJ 356:j84

7. Riens B, Erhard M, Mangiapane S (2012) Arztkontakte im Jahr 2007. Versorgungsatlas-Bericht, Bd. 12/02. Zentralinstitut für die kassenärztliche Versorgung in Deutschland (Zi), Berlin https://doi. org/10.20364/VA-12.02

8. Grobe TG, Steinmann S, Szecsenyi J (2017) Arztreport 2017. Schriftenreihe zur Gesundheitsanalyse, Bd. 1. Asgard, Siegburg

9. o.V. Bagatellfälle blockieren Notaufnahmen. Süddeutsche Zeitung vom 06. September 2016

10. Schreyögg J et al (2014) Endbericht, Forschungsauftrag zur Mengenentwicklung nach $\S 17 \mathrm{~b}$ Abs. 9 KHG. http://www.dkgev.de/media/file/ 17192.2014-07-10_Anlage_Forschungsberichtzur-Mengenentwicklung_FIN.pdf. Zugegriffen: 31. Juli 2017

11. Schoen Cet al (2011) New 2011 survey of patients with complex care needs in eleven countries finds that care is often poorly coordinated. Health Aff 30(12):2437-2448
12. National Center for Biotechnology Information (2017) https://www.ncbi.nlm.nih.gov/. Zugegriffen:31. Juli 2017

13. ResearchGate GmbH (2017) https://www. researchgate.net/home. Zugegriffen: 31. Juli 2017

14. Bynum JP, Bernal-Delgado E, Gottlieb D, Fisher E (2007) Assigning ambulatory patients and their physicians to hospitals: a method for obtaining population-based provider performance measurements. Health Serv Res 42:45-62

15. Landon BE et al (2013) Med Care 51(8):715-721

16. Stukel TA et al (2013) Multispecialty physician networks in Ontario. Open Med 7(2):e40-e55

17. Pham HHet al (2009) Primary care physicians' links to other physicians through medicare patients: the scope of care coordination. Ann Intern Med 150:236-242

18. Pollack CE et al (2012) Patient sharing among physicians and costs of care: a network analytic approach to care coordination using claims data. J Gen Intern Med 28(3):459-465

19. Hussain T et al (2015) Collaboration between surgeons and medical oncologists and outcomes for patients with stage III colon cancer. J Oncol Pract 11(3):e388-e397

20. Diestel R (2016) Graphentheorie, 5. Aufl. Springer, Heidelberg

21. Carrington PJ, Scott J, Wasserman S (Hrsg) (2005) Models and methods in social network analysis. Cambridge University Press, New York

22. Newman MEJ (2015) Networks an introduction. Oxford University Press, Oxford, S 168-193

23. Newman MEJ (2006) Modularity and community structure in networks. Proc Natl Acad Sci USA 103(23):8577-8582

24. Moen EL et al (2016) An Analysis of Patient-Sharing Physician Networks and Implantable Cardioverter Defibrillator Therapy. Health Serv Outcomes Res Methodol 16(3):132-153

25. Robins $\mathrm{G}$ et al (2007) Recent developments in exponential random graph $\left(p^{*}\right)$ models for social networks. Soc Netw 29(2):192-484

26. Fortunato $S$ (2010) Community detection in graphs. Phys Rep 486(2010):75174

27. Yang Z, Algesheimer R, Tessone C (2016) A comparative analysis of community detection algorithms on artificial networks. Sci Rep 6:30750. https://doi.org/10.1038/srep30750

28. Landon BE et al (2012) Variation in patient-sharing networks of physicians across the United States. JAMA 308(3):265-273

29. Barnett ML et al (2012) Physician patient-sharing networks and the cost and intensity of care in US hospitals. Med Care 50(2):152-160

30. Carson MB et al (2016) An outcome-weighted network model for characterizing collaboration. PLOSONE 11(19):e163861

31. Stillfried D, Czihal T (2014) Welchen Beitrag liefern funktional definierte Populationen zur Erklärung regionaler Unterschiede in der medizinischen Versorgung? Bundesgesundheitsblatt Gesundheitsforschung Gesundheitsschutz 57:197-206

32. Wennberg J (2010) Tracking medicine. Oxford University Press, Oxford

33. Riens B, Bätzing-Feigenbaum J (2009) Leitliniengerechte Therapie bei Herzinsuffizienz. Versorgungsatlas-Bericht, Bd. 14/03. Zentralinstitut für die kassenärztliche Versorgung in Deutschland (Zi), Berlin https://doi.org/10.20364/VA-14.03

34. Zentralinstitut für die kassenärztliche Versorgung in Deutschland (2014) Herzinsuffizienz - mit leitliniengerechter Versorgung nicht schlapp machen. http://www.zi.de/cms/veranstaltungen/ zi-forum/juni-2014/ (Erstellt: 4. Juni 2014). Zugegriffen: 31. Juli 2017

35. Bynum J et al (2010) Measuring racial disparities in the quality of ambulatory diabetes care. Med Care 48(12):1057-1063

36. von Stillfried D, Schulz M, Bätzing-Feigenbaum $J$ (2016) Herausforderungen für die ambulante Versorgung Demenzkranker - ein Vergleich der Regionen. https://www.kvb.de/fileadmin/ kvb/dokumente/UeberUns/Termine/2016/Tagder-Versorgungsforschung/KVB-Termin-TagVersorgungsforschung-2016-Vortrag-Stillfried. pdf. Zugegriffen: 31. Juli 2017 (Vortrag Tag der Versorgungsforschung der KV Bayerns [München])

37. Koller D, Vogt V, Sundmacher L (2015) Intersectoral networks in Germany, or, am I part of the right club? Wennberg International Collaborative WIC Policy Conference, Berlin, 04.-05.06.2015.

38. Schang L, Koller D, Sundmacher L (2017) Accountable Care in Deutschland? Sektorenübergreifende Qualitätsmessung auf Basis intersektoraler Netzwerke von Krankenhäusern und Vertragsarztpraxen. 9. DGGÖ Jahrestagung, Basel, 09.-10.03.2017.

39. Stukel T (2016) Ontario health links: an organizational policy initiative that targets high need, high cost patients. Herbsttagung der Wennberg International Collaborative (WIC), Oxford, 12.-14.06.2016. (http:// wennbergcollaborative.org/uploads/documents/ PublicPresentationMaterials.pdf, 220-266. Zugegriffen:31. Juli 2017)

40. Gemeinsamer Bundesausschuss (2017) https://innovationsfonds.g-ba.de/projekte/ versorgungsforschung/acd-accountablecare-in-deutschland-verbesserung-derpatientenversorgung-durch-vernetzung-vonleistungserbringern-und-informierten-dialog. 45 . Zugegriffen: 31. Juli 2017

41. Vertragsärztliche Abrechnungsdaten gemäß§ 295 SGB V, Arzneimittelabrechnungsdaten gemäß $\S 300$ Abs. 2 SGBV

42. Zentralinstitut für die kassenärztliche Versorgung in der Bundesrepublik Deutschland (Zi) (2017) versorungsatlas.de - Angewandte Methoden. http:// www.versorgungsatlas.de/der-versorgungsatlas/ angewandte-methoden.Zugegriffen:31. Juli 2017

43. Kassenärztliche Bundesvereinigung (2017) Einheitlicher Bewertungsmassstab (EBM). http:// www.kbv.de/html/ebm.php. Zugegriffen: 31 . Juli 2017

44. Kraut A (2013) Klinisch-epidemiologische Forschung anhand von Daten der gesetzlichen Krankenkassen - Grenzen und Möglichkeiten. Dissertation zur Erlangung des akademischen Grades Doktorin Public Health (Dr. P.H.) Universität Bremen. https:// www.deutsche-digitale-bibliothek.de/binary/ GQN5GBIZK7VILATYGJN67MPXW044OJPV/full/1. pdf.Zugegriffen:31. Juli 2017

45. Riens B, Bätzing-Feigenbaum J (2014) Leitliniengerechte Therapie bei Herzinsuffizienz (2009). Versorgungsatlas-BerichtNr. 14/03.Zentralinstitut für die kassenärztliche Versorgung, Berlin. https:// doi.org/10.20364/VA-14.03

46. Girvan M, Newman MEJ (2002) Community structure in social and biological networks. Proc Natl Acad Sci USA 99:7821-7826

47. PonsP,LatapyM(2005)Computing communities in large networks using random walks. https://arxiv. org/pdf/physics/0512106v1.pdf. Zugegriffen: 31 . Juli 2017

48. Clauset A, Newman MEJ, Moore C (2004) Finding community structure in very large networks. 
https://arxiv.org/pdf/cond-mat/0408187.pdf. Zugegriffen:31.Juli 2017

49. Sudhakar-Krishnan V, Rudolf M (2007) How important is continuity of care? Arch Dis Child 92(5):381-383

50. Barker I, Steventon A, Deeny SR (2017) Association between continuity of care in general practice and hospital admissions for ambulatory care sensitive conditions: cross sectional study of routinely collected, person level data. BMJ 356:j84

51. Vogt $V$, Sundmacher $L$ (2016) Continuity of care in the ambulatory sector and hospital admissions among patients with heart failure in Germany. Eur J Public Health. https://doi.org/10.1093/eurpub/ ckw018

52. Drösler S et al (2016) Möglichkeiten der Berücksichtigung von Besonderheiten der Versorgungsstrukturen als modifizierender Faktor des risikoadjustierten Behandlungsbedarfs im Rahmen der vertragsärztlichen Vergütung. Gutachten im Auftrag der Kassenärztlichen Vereinigung Bayerns (KVB). http://www.zi.de/ $\mathrm{cms/fileadmin/images/content/Gutachten/KVB-}$ Gutachten_Versorgungsstrukturen_2016-02-18. pdf.Zugegriffen:31. Juli 2017

53. Albrecht M (2015) Ambulantes Potential in der stationären Notfallversorgung. Gutachten für das Zentralinstitut für die kassenärztliche Versorgung in Deutschland. http://www.zi.de/cms/fileadmin/ images/content/Gutachten/Zi-IGES-Gutachten Ambulantes_Potential_Notf\%C3\%A4lle_Teil_1_ 2015-03-19.pdf.Zugegriffen:31. Juli 2017

54. Watson DE et al (2005) Planning for renewal: mapping primary health care in British Columbia. Centre for Health Services and Policy Research, University of British Columbia

55. Horenkamp-Sonntag D (2017) Versorgungsforschung und Qualitätsmessung mit GKV-Routinedaten. Die Belastbarkeit von Abrechnungsdaten der gesetzlichen Krankenversicherung für die wissenschaftliche Nutzung. Nomos, Baden-Baden 